Draft VERsion June 3, 2021

Preprint typeset using $\mathrm{LT}_{\mathrm{E}} \mathrm{X}$ style emulateapj v. 12/16/11

\title{
HAFFNER 16 REDUX: RE-VISITING A YOUNG CLUSTER IN THE OUTER GALAXY ${ }^{12}$
}

\author{
T. J. DAVIDGE \\ Dominion Astrophysical Observatory, \\ National Research Council of Canada, 5071 West Saanich Road, \\ Victoria, BC Canada V9E 2E7 \\ tim.davidge@nrc.ca \\ Draft version June 3, 2021
}

\begin{abstract}
Images and spectra recorded with the Gemini Multi-Object Spectrograph on Gemini South are used to investigate the stellar content of the open cluster Haffner 16 . The $\left(i^{\prime}, g^{\prime}-i^{\prime}\right)$ color-magnitude diagram (CMD) constructed from these data extends over 10 magnitudes in $i^{\prime}$, sampling the cluster main sequence (MS) and 5 magnitudes of the pre-MS (PMS). The fraction of unresolved equal mass binaries among PMS stars is estimated to be $0.6 \pm 0.1$. The isochrones do not track the PMS on the CMD, in the sense that the PMS has a shallower slope on the CMD than predicted by the models. Still, a dip in star counts that is associated with the relaxation of PMS stars onto the MS is identified near $i^{\prime}=17$. The depth and brightness of this feature - as well as the morphology of the cluster MS on the CMD - are matched by models with a slightly sub-solar metallicity that have an age $\sim 20$ Myr and a distance modulus of $12.3 \pm 0.2$. A light profile of Haffner 16 is constructed in the W1 filter $\left(\lambda_{c e n}=3.4 \mu \mathrm{m}\right)$ which suggests that the cluster is surrounded by a diffuse stellar halo. Spectra are presented of candidate cluster MS and PMS stars selected according to location on the CMD. The spectra show characteristics that are suggestive of a sub-solar metallicity. $\mathrm{H} \alpha$ emission is common among objects on the PMS locus on the CMD near $i^{\prime}=18$. It is suggested that the location of the Haffner 16 PMS on the CMD is affected by large-scale cool spot activity, likely induced by rapid stellar rotation.
\end{abstract}

Subject headings: open clusters and associations: individual (Haffner 16)

\section{INTRODUCTION}

Star clusters play an important role as basic astrophysical calibrators. It is then ironic that the clusters that are seen today are likely the survivors of a process (or processes) that disrupt(s) the vast majority of stellar groupings early in their lifetimes (e.g. Lada \& Lada 2003); hence, the clusters that provide constraints on say - stellar properties may not be typical of the environments where the majority of stars in the Galaxy formed. A likely cause of early cluster disruption is the rapid outflow of gas driven by massive stars, which changes the cluster potential (e.g. Matzner \& Jumper 2015). For those few clusters that survive the early loss of mass, the pace of subsequent dynamical evolution may depend on factors such as environment (e.g. Kruijssen et al. 2012; Silva-Villa et al. 2014), galaxy morphology (e.g. de Grijs et al. 2013), and the properties of the molecular clouds from which they form (Fujii \& Portegies Zwart 2016). Still, despite the diverse range of potential factors that might play a role in cluster disruption, a census of star clusters that spans a range of ages and masses in nearby

\footnotetext{
${ }^{1}$ Based on observations obtained at the Gemini Observatory, which is operated by the Association of Universities for Research in Astronomy, Inc., under a cooperative agreement with the NSF on behalf of the Gemini partnership: the National Science Foundation (United States), the National Research Council (Canada), CONICYT (Chile), Ministério da Ciência, Tecnologia e Inovação (Brazil) and Ministerio de Ciencia, Tecnología e Innovación Productiva (Argentina).

2 This research has made use of the NASA/IPAC Infrared Science Archive, which is operated by the Jet Propulsion Laboratory, California Institute of Technology, under contract with the National Aeronautics and Space Administration.
}

galaxies reveals that cohorts of coeval clusters are whittled down uniformly by a factor of $\sim 6$ in total number per decade in age (Fall \& Chandar 2012).

The timescale for the evolution of massive stars is a few Myr. Clusters with ages that are less than a few tens of Myr are thus of interest for studies of cluster evolution, as they sample a phase when systems that survived gas removal are in the early stages of any subsequent evolution. Comparing the properties of many such systems may then provide clues into the properties of clusters that survive early disruption.

Haffner 16 is a young cluster in the outer Galaxy that has not been extensively studied in the past. Vogt \& Moffat (1972) present a CMD with a main sequence (MS) that is populated by OB stars, thereby pointing to a young age. McSwain \& Gies (2005) included Haffner 16 in an investigation of bright emission line sources in young clusters. They construct a CMD that goes as faint as $y \sim 17.5$ and assign an age of 12 Myr. This age was estimated from stars that are distributed over a much wider field than the central regions of the cluster, and so it may be biased by non-cluster members. A single Be star candidate was found out of a sample of 19 B stars studied.

Davidge et al. (2013) used deep adaptive optics (AO)-corrected near-infrared (NIR) images to examine the stellar content of Haffner 16 . An age $\leq 10$ Myr was found from the $K$ magnitude of the MS cut-off (MSCO), which is the point on the CMD below which stars are still evolving on the pre-MS (PMS). A distance modulus of 13.5 was assumed. Davidge et al. (2013) also discuss photometry obtained from narrow-band images that suggests that many stars with $K \geq 15$ in Haffner 16 have 
$\mathrm{Br} \gamma$ in emission, suggesting that chromospheric activity may be common among low mass stars in the cluster.

Haffner 16 was selected for investigation by Davidge et al. (2013) in part because existing data suggested that it had a compact size that would fit within the $\sim 85$ arcsec science field of the multi-conjugate $\mathrm{AO}$ system used in that study. However, Davidge et al. (2013) found that PMS stars in Haffner 16 are distributed over a larger area on the sky than the brightest cluster members, raising the possibility that Haffner 16 might be larger than originally thought. Deep photometric and spectroscopic observations of stars in and around Haffner 16 that cover many arcminutes on the sky may thus provide additional insights into its basic properties. Wide field observations are also of interest because stars that are no longer bound to a cluster may linger near it for some time (Pfalzner et al. 2014). This raises the possibility of being able to reconstruct the primordial mass function (i.e. the mass function prior to disruption) of a cluster even if there has been significant dynamical evolution and the cluster is in the process of dissolving.

In the present paper, deep imaging and spectra of Haffner 16 that were recorded with the Gemini MultiObject Spectrograph (GMOS) on Gemini South are discussed. The CMD and luminosity function (LF) constructed from the images sample sources with masses as low as a few tenths solar. In addition to measuring age and reddening, the CMD was also used to select objects for follow-up spectroscopy. Multi-slit spectra were obtained of 92 sources with a common spectral coverage of $0.53-0.84 \mu \mathrm{m}$, although the spectra of individual stars extend beyond these wavelength limits.

The paper is structured as follows. The imaging and spectroscopic observations are described in Section 2, as are the procedures that are used to remove instrumental and atmospheric signatures from the raw data. The photometric measurements are the subject of Section 3, while the cluster light profile constructed from infrared images is presented in Section 4. The light profile is used to identify areas on the sky that are likely dominated by cluster stars, which is an important issue for Haffner 16 given the high density of field stars. The CMD and $\mathrm{LF}$ are examined in Sections 5 and 6, while spectra of individual stars are discussed in Section 7. The paper closes in Section 8 with a summary and discussion of the results.

\section{OBSERVATIONS \& REDUCTIONS}

The images and spectra that are the basis of this study were recorded with GMOS (Hook et al. 2004) on Gemini South as part of program GS-2014A-Q-84 (PI: Davidge). GMOS is the facility visible-light imager and spectrograph. The detector was 1 a mosaic of three $2048 \times$ 4068 EEV CCDs. Each $13.5 \mu \mathrm{m}$ square pixel subtended 0.073 arcsec on the sky. The three CCDs covered an area that is larger than that illuminated by the sky as spectra may be dispersed outside of the sky field. The images and spectra were both recorded with $2 \times 2$ pixel binning.

\subsection{GMOS Images}

1 The CCDs that make up the GMOS detector have since been replaced.
TABLE 1

GMOS IMAGES

\begin{tabular}{cll}
\hline Filter & $\begin{array}{l}\text { Exposures } \\
(\mathrm{sec})\end{array}$ & $\begin{array}{l}\text { FWHM } \\
(\operatorname{arcsec})\end{array}$ \\
\hline$g^{\prime}$ & $1 \times 1$ & 0.55 \\
& $1 \times 100$ & 0.55 \\
$i^{\prime}$ & $1 \times 1$ & 0.45 \\
& $5 \times 20$ & 0.45 \\
\hline
\end{tabular}

$g^{\prime}$ and $i^{\prime}$ images of Haffner 16 were recorded on the night of December 31, 2013. Exposure times and image quality measurements are summarized in Table 1. Long and short exposures were recorded in both filters so that a CMD that samples stars over a large range of brightnesses could be constructed.

A series of calibration frames were also obtained. Biases were recorded at the end of the night, and these were median-combined to construct a final bias frame. A fringe frame in $i^{\prime}$ and flat field frames constructed from observations of the twilight sky were provided by Gemini as part of the calibration package for this program.

A standard processing flow for CCD mosaic imaging was applied to remove instrumental signatures from the images. To start, the output from each CCD was multiplied by its gain, and this was followed by bias subtraction, the trimming of overscan regions, and division by a flat-field frame. The fringe frame, scaled to match the exposure time of the images, was then subtracted from the $i^{\prime}$ images.

The deep $i^{\prime}$ images were recorded with on-sky dithering. These were aligned using stars across the science field as reference points and averaged together after processing. All other exposures were shifted to match the reference frame defined by the final deep $i^{\prime}$ image, which is shown in Figure 1. The GMOS science field is divided into four regions to facilitate the analysis of the photometry and spectra (Section 4), and the boundaries are indicated in Figure 1.

\subsection{GMOS Spectra}

The spectra were recorded during five nights in March and April 2014. The primary criterion for target selection was location on the $\left(i^{\prime}, g-i\right)$ CMD. High priority was assigned to CMD-selected sources in the middle CCD of the mosaic to facilitate common wavelength coverage. Masks were designed from images in which the cluster was centered on this CCD (Figure 1), and so this detector is also where the density of cluster members is highest. Radial velocities measured from these data are of little use for assessing cluster membership given the modest resolution of the spectra.

Six slit masks were designed, with the target stars in each mask having similar magnitudes. The date that each mask was observed, the total exposure time, and the FWHM of stars in the slits are listed in Table 2. The exposure times reflect the brightnesses of the stars targeted for each mask.

The spectra were dispersed with the R400 grating $\left(\lambda_{\text {blaze }}=7640 \AA, 400\right.$ lines $\left./ \mathrm{mm}\right)$, with a GG455 filter deployed to suppress light from higher orders. Each mask was observed with two grating rotations such that the central wavelengths for a source at the center of the detector mosaic is either 6750 or $6800 \AA$. Having spectra 


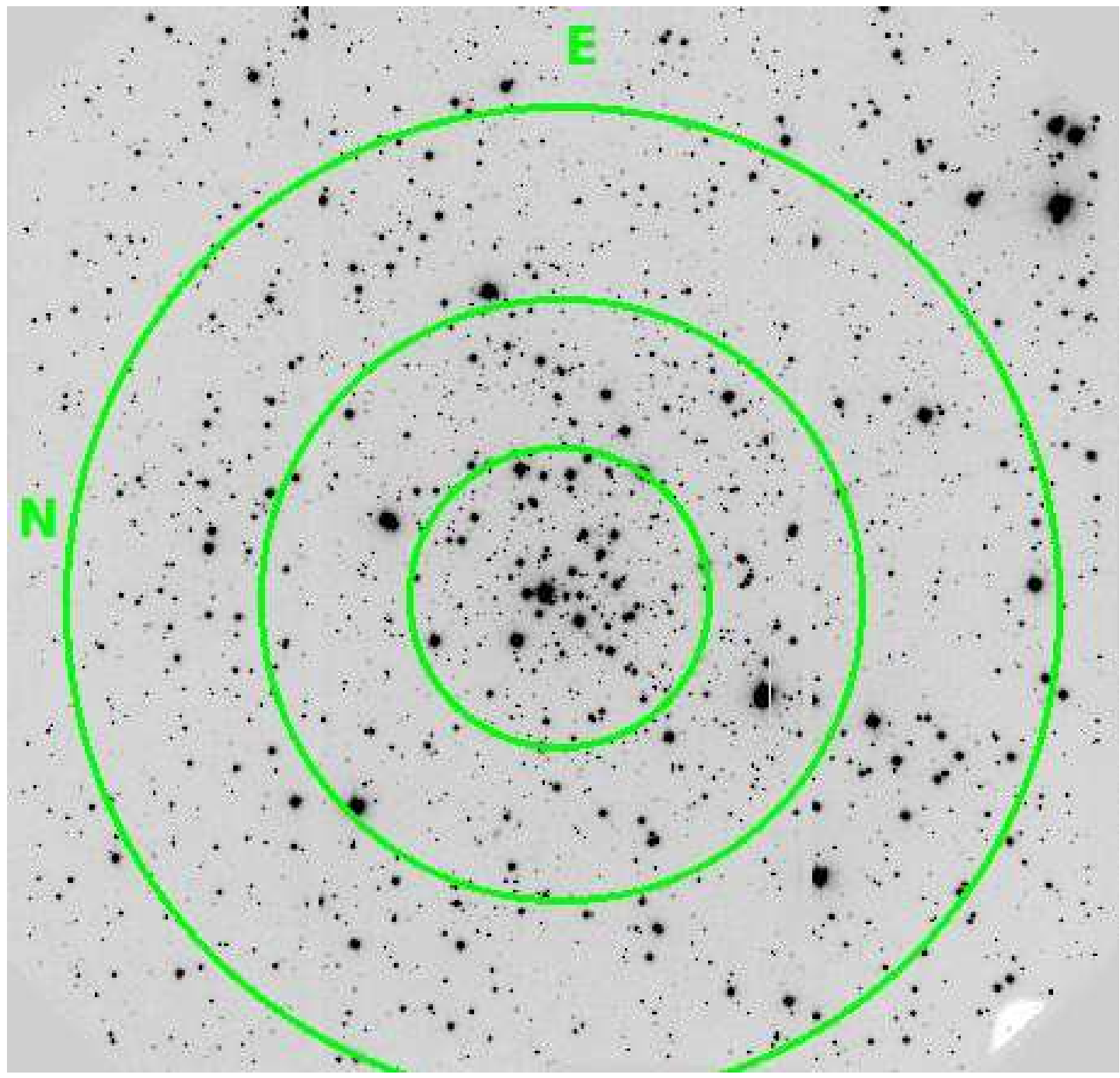

FIG. 1.- Deep $i^{\prime}$ image of Haffner 16. The image covers $5.9 \times 5.6$ arcmin, with North to the left, and East at the top. The green circles mark the boundaries of the regions that are used in the analysis of the photometric and spectroscopic observations. Working outwards from the cluster center, these are the Center, Shoulder, Field 1, and Field 2 regions.

TABLE 2

GMOS SPECTRA

\begin{tabular}{clcc}
\hline $\begin{array}{c}\text { Mask } \\
\#\end{array}$ & $\begin{array}{l}\text { UT Date } \\
\text { Observed } \\
(2014)\end{array}$ & $\begin{array}{c}\text { Exposure } \\
\text { Time } \\
\text { (secs) }\end{array}$ & $\begin{array}{c}\text { FWHM } \\
\text { (arcsec) }\end{array}$ \\
\hline 1 & April 3 & $2 \times 150$ & 1.0 \\
2 & March 19 & $2 \times 150$ & 0.9 \\
3 & March 30 & $2 \times 600$ & 1.0 \\
4 & March 27 & $2 \times 600$ & 0.9 \\
5 & April 3 & $4 \times 600$ & 0.9 \\
6 & April 2 & $4 \times 600$ & 0.9 \\
\hline
\end{tabular}

with two wavelength settings for each mask allows the gaps between the GMOS CCDs to be filled during processing.

With the exception of acquisition stars (see below), stars selected for spectroscopy were placed in $1 \times 5$ arcsec slitlets. In the vast majority of cases the star was positioned near the center of the slit, as measured along the spatial direction. However, if another star could be placed in the slit with only modest re-positioning of the slit then the mask design was adjusted to allow for this. As these additional stars were selected solely based on their proximity to a primary target, they are a serendipitous sample of disparate objects (Section 7.4).

The on-sky alignment of each mask was checked using three stars that are centered in $2.4 \times 2.4$ arcsec apertures. While the primary purpose of these stars is mask centroiding, spectra of these objects were also recorded. The alignment stars were selected based on brightness, position in the CMD, and location near the edge of the science field. As these objects are placed in a wider aperture than the majority of objects then their spectral resolution is defined by the seeing disk. However, there is no discernable difference between the absorption line profiles of sources in slitlets and those in the acquisition apertures. This is likely because the seeing when the spectra were recorded was comparable to the 1 arcsec slitlet width (Table 2 ).

The alignment stars tend to be among the brightest objects in the spectroscopic sample. The restrictions on brightness, position on the CMD, and location in the field (near the edge of the science field) limits the number of available alignment stars. Hence, some alignment stars were used in multiple masks, with the result that more than one spectrum was recorded of some objects. 
Calibration frames were also recorded. A frame that monitors the fixed bias pattern was constructed from bias observations that were obtained within a few weeks of the spectra. Images that monitor pixel-to-pixel variations in sensitivity and variations in throughput along the slitlets ('flats') were recorded for each mask midway through the on-sky observing sequence. The light source for the flats was a continuum lamp in the Gemini Calibration Unit. Spectra of a CuAr emission source ('arcs') were recorded for each mask at both wavelength settings. Arcs were typically recorded at the end of the night or during the following day.

The processing of the spectra proceeded as follows. The signal from the three CCDs were multiplied by their respective gains. The results were bias-subtracted and the overscan was trimmed from each CCD. The biassubtracted frames were averaged together after shifting to adjust for wavelength offsets, with emission lines in the arc spectra serving as a reference. Signal in the gaps between the detectors was ignored when combining the frames. Cosmic-rays were identified using an edgesensing algorithm, and then removed by interpolating the signal from surrounding pixels. Bad columns and hot pixels were also suppressed by interpolating over the affected areas. The flat-field and arc frames were processed in the same way as the science frames.

Slitlets were extracted from the co-added exposures for subsequent processing. The signal in each slitlet was divided by a normalized flat-field frame that was constructed for that slitlet. Wavelength calibration was then done using bright arc lines as reference points. Each wavelength-calibrated two-dimenional spectrum was skysubtracted by taking the mean sky level at both ends of the slit on a row-by-row basis. If more than one source was in the slit then the sky level was measured at a location along the slit where stellar contamination was lowest.

Individual stellar spectra were extracted from the sky-subtracted slitlets by co-adding the signal within the FWHM of each stellar profile. The wavelength response of each spectrum at this stage of processing contains contributions from the atmosphere, the telescope, GMOS optics, the detector, and the flat-field light source. These contributions were removed by dividing each Haffner 16 spectrum by the spectrum of the white dwarf LTT 4364, which was observed by GMOS in long-slit mode with the same grating, central wavelengths, slit width, and ordersorting filter as the cluster spectra. The spectrum of LTT 4364 was processed in the same manner as the Haffner 16 spectra, and division by this spectrum normalized the cluster spectra to that of LTT 4364. Telluric absorption features were also suppressed. Residual variations in wavelength response were then removed by fitting a low order continuum function to each extracted spectrum.

\section{STELLAR PHOTOMETRY}

Photometric measurements were made with the point-spread function (PSF)-fitting program ALLSTAR (Stetson \& Harris 1988). The star lists, PSFs, and preliminary brightnesses used by ALLSTAR were generated by running tasks in the DAOPHOT (Stetson 1987) package. A single PSF for each image was constructed by combining the signal from bright, isolated, unsaturated stars located across the imaged field. Haffner 16 is at low
Galactic latitudes, and so there is a pervasive population of faint objects, some of which inevitably fall within or just outside of the extraction radius of the PSF stars. These contaminants were subtracted out using progressively improved versions of the PSF.

The brightnesses of the vast majority of objects were measured by fitting the PSF to the central regions of the stellar profiles. However, there is a modest number of bright sources that are saturated near their profile centers, even in the short exposure images. PSF-fitting was done in the PSF wings of these objects.

The photometry was calibrated using observations of standard stars in the 075944-59550 field. The standard stars were observed with GMOS on the night of December 30, 2013 (i.e. the night before the images for Haffner 16 were recorded). Magnitudes for the standard stars in the SDSS system are listed in the Southern Standard Stars for the u'g'r'i'z' System website 2 .

\section{THE MIR LIGHT PROFILE}

There is a high density of field stars near Haffner 16 , and contamination from these objects have the potential to affect an investigation of cluster properties. However, the light profile of Haffner 16 can be used to identify radial intervals where the light is dominated by cluster members. In this section we discuss the light profile of Haffner 16 constructed from wide-field images that were recorded as part of the Wide-Field Infrared Explorer (WISE) All-Sky survey (Wright et al. 2010). Images taken with WISE cover wavelengths that are less susceptible to dust absorption than those at visible or NIR wavelengths and - at least at the short wavelength range of WISE coverage - sample light that is predominantly photospheric in origin.

Processed ALL-Sky survey images were downloaded from the WISE archive [3. Processed images in this part of the sky are restricted to the W1 $\left(\lambda_{\text {cen }}=\right.$ $3.4 \mu \mathrm{m})$ and $\mathrm{W} 2\left(\lambda_{c e n}=4.6 \mu \mathrm{m}\right)$ filters. The $\mathrm{W} 1$ filter samples wavelengths where the contribution from photospheric light is expected to dominate the signal, and so the light profile was constructed from observations in this filter.

The azimuthal averaging technique described by Davidge et al. (2016) was used to multiplex the signal from cluster stars and suppress bright field stars. In brief, the image is divided into 24 azimuthal zones centered on the cluster. The mean signal at each radius is found within each zone. There are then 24 measurements of the surface brightness at a given radius, and the median of these is adopted as the cluster surface brightness at that radius. A basic assumption is that the cluster light follows circular isophotes, and the distribution of bright stars near the center of Haffner 16 in Figure 1 suggests that this assumption is reasonable. A $26 \times 26$ arcsec median filter was applied to the images prior to combination to suppress light from bright stars, while retaining the light from the numerous unresolved stars that make up the main body of the cluster.

The light profile of Haffner 16 in the W1 filter is shown in Figure 2. The W1 surface brightness measurements were calibrated using zeropoints from Jarrett et

\footnotetext{
2 http://www-star.fnal.gov

3 http://wise2.ipac.caltech.edu/docs/release/allsky/
} 
al. (2011). Background light, which was measured at distances in excess of 5 arc minutes from the cluster center, is the dominant source of uncertainty in the profile at large radii. The level of this uncertainty is illustrated with the green lines in the figure, which show the range in surface brightness measurements that result if the sky level is varied by $\pm 1 \sigma$, where the dispersion was estimated from sky level measurements made in different sub-sections of the field at large offsets from Haffner 16.

The light distribution in Figure 2 is more-or-less flat within 50 arcsec of the cluster center, and then drops over a 40 arcsec interval. The background-subtracted light level at large radii does not drop to zero in the area imaged with GMOS, indicating that there is an over-density of light with respect to larger radii throughout the GMOS science field. Diffuse stellar halos have been detected around other young clusters (e.g. Davidge 2012), and some of the stars in the circumcluster area may be present or former cluster members, or may have formed at a similar time as the main body of the cluster. Indeed, stars that are no longer bound to a cluster may linger near it for an extended period of time (Pfalzner et al. 2015), thus forming a halo. The collapse of a molecular cloud may involve the formation of both a cluster and a diffuse population of surrounding objects (e.g. Bonnell et al. 2011), also resulting in a halo.

For the purpose of the current investigation, the analysis of cluster stars is restricted to $\leq 90$ arcsec from the center of Haffner 16. Stars at larger radii are deemed to belong to a 'field' population. While cluster members are almost certainly present at larger radii, the scatter in the CMDs presented in Section 5 indicates that noncluster stars dominate at distances in excess of 90 arcsec from the center of Haffner 16.

\section{THE COLOR-MAGNITUDE DIAGRAM}

The CMDs of stars in the four radial intervals indicated in Figure 2 are shown in Figure 3. The CMDs in the two left hand panels are of regions where the integrated light is dominated by cluster stars, while the CMDs in the other two panels cover radii where noncluster stars are expected to dominate (Section 4). The points in the CMDs with $i^{\prime}<17.5$ were obtained from the short exposure images; those with $i^{\prime}<14$ were obtained by fitting the PSF to the wings of stellar profiles to avoid the saturated profile centers. All points with $i^{\prime}>17.5$ were measured from the long exposure images.

Stars in Haffner 16 define a tight locus of objects in the CMDs of the Center and Shoulder regions. A well-defined blue MS is evident at $i^{\prime}<16$ in the Center region CMD. Cluster MS stars may also be present in modest numbers in the CMDs of the Shoulder and Field 1 regions, although the scatter near the bright end of those CMDs suggests greater fractional contamination from non-cluster stars than at smaller radii. The PMS forms a tight line in the Center and Shoulder CMDs that runs from $i^{\prime} \sim 18$ to $i^{\prime} \sim 21.5$, where it terminates at the faint limit of the CMD near $g^{\prime}-i^{\prime} \sim 3$. The GMOS images thus sample stars in Haffner 16 that are as faint as $g^{\prime} \sim 24.5$.

The green line in each panel is a hand-drawn representation of the cluster locus. The upper part of the cluster fiducial is based mainly on the Center CMD, whereas at fainter magnitudes it relies on both the Center and
Shoulder region CMDs. The CMD of Haffner 16 between $i^{\prime}=17$ and 18 is not well populated (Section 6), and so the cluster sequence may be poorly defined at those magnitudes.

Given the Galactic latitude of Haffner 16, it is not surprising that the Field 1 and 2 CMDs are well populated. The substantial scatter among the stars with $i^{\prime}>15$ in the Field 1 and 2 CMDs is consistent with the majority of the bright stars in these regions being foreground/background objects. As for the faint end, there is a clump of objects in the Field 1 and 2 CMDs with colors that are similar to those of PMS stars in Haffner 16. The nature of these objects is unclear, and their presence raises concerns about the ability to distinguish between faint cluster and non-cluster members.

While identifying individual cluster stars may be problematic (although in Section 7 it is shown that stars with $i^{\prime}$ between 18 and 19 that fall on the middle CCD of the GMOS detector mosaic tend to have spectroscopic characteristics that are suggestive of cluster membership), the spatial distribution and photometric properties of PMS stars in Haffner 16 as an ensemble can be characterized statistically near the faint end of the CMD. This is demonstrated in Figure 4, where the $g^{\prime}-i^{\prime}$ color distributions near the faint end of the CMDs are shown. Each panel in Figure 4 shows stellar densities in \pm 0.25 magnitude bins centered on different $i^{\prime}$ magnitudes. The color distributions that remain after subtracting stellar densities in Fields 1 and 2 from the densities in the Cluster and Shoulder regions - and so should characterize a cluster population that is free of contamination by noncluster stars - are shown in the right hand panel.

The color distributions in the right hand panel contain a population of objects with red $g^{\prime}-i^{\prime}$ colors that remains at each $i^{\prime}$ after subtracting the star counts in Fields 1 and 2. This residual population is made up of PMS objects in Haffner 16. The broad width of the PMS $g^{\prime}-i^{\prime}$ distribution is likely the result of a number of factors, including binarity (see below), a dispersion in the intrinsic photometric properties of the PMS objects, and the \pm 0.25 magnitude binning in $i^{\prime}$ that was imposed to obtain statistically significant numbers of sources. Objects with $g^{\prime}-i^{\prime}<2$ at each $i^{\prime}$ in the cluster color distribution are suppressed when the Field 1 and 2 counts are subtracted from the cluster counts. This indicates that these objects have a uniform distribution on the sky, as expected for stars that are not cluster members.

\subsection{The Frequency of Equal Mass Binaries}

The red line in Figure 3 is the cluster fiducial shifted upwards by 0.75 magnitudes to mark the expected location in the CMD of unresolved equal mass binaries. Processes other than binarity (e.g. star spots) may cause cluster members to fall near the binary sequence. However, stars near the binary sequence are seen in the Center and Shoulder CMDs over a large range of magnitudes, and hence over a range of evolutionary states and effective temperatures. This is difficult to explain if these sources are not binaries, and so in the remainder of the paper these objects are referred to as unresolved equal mass binaries.

Source counts were made between $i^{\prime}=18$ and 20 in a strip about the red sequence with a width \pm 0.2 magnitude in $g^{\prime}-i^{\prime}$ to determine if unresolved equal mass bi- 


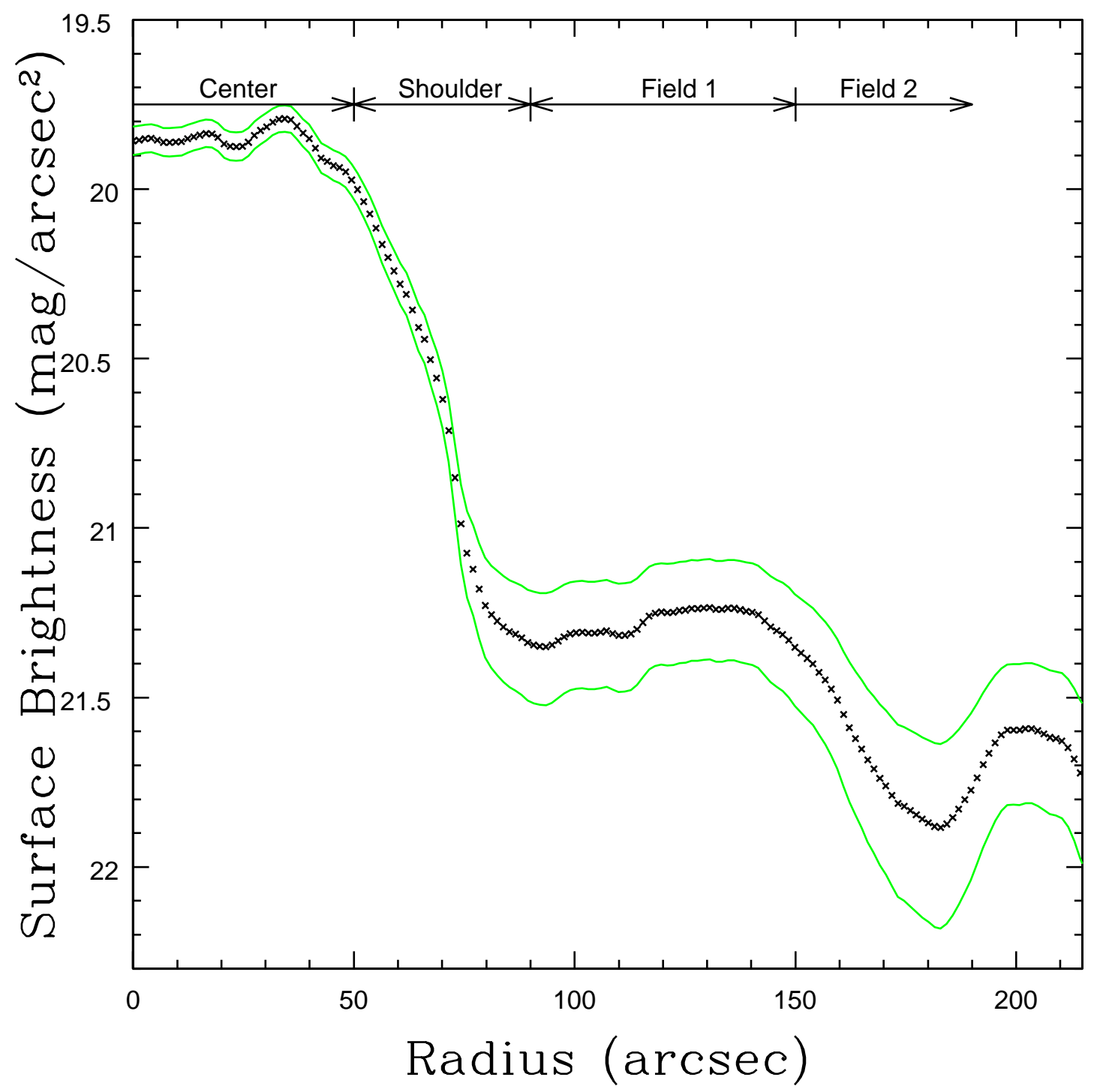

FIG. 2.- Light profile of Haffner 16 in the W1 $\left(\lambda_{c e n}=3.4 \mu \mathrm{m}\right)$ filter. The profile was constructed using the procedure described in the text. The green lines show the surface brightnesses that result if the sky level is varied by $\pm 1 \sigma$, with the dispersion computed from sky level measurements made at different locations. The radial intervals that are used in the analysis of the photometry and spectra are indicated.

naries in Haffner 16 occur in measureable numbers. This magnitude range samples the upper regions of the cluster PMS, and was selected because it is where the binary sequence is well offset from the single star sequence. The densities of objects near the red sequence in this part of the CMD are $5.6 \pm 1.6 \operatorname{arcmin}^{-2}$ (Center), $4.7 \pm 1.0$ $\operatorname{arcmin}^{-2}$ (Shoulder), and $1.4 \pm 0.2 \operatorname{arcmin}^{-2}$ (Field 1 and 2 combined). Thus, there is a statistically significant population of objects in the Center and Shoulder regions near the binary sequence that is not present in Fields 1 and 2 .

The frequency of unresolved equal mass binaries in Haffner 16 can be estimated after measuring the number of stars along the single star sequence. The density of sources with $i^{\prime}$ between 18 and 20 that are within \pm 0.2 magnitude of the green (i.e. single star) fiducial in the Center and Shoulder region is $9.5 \pm 1.2 \operatorname{arcmin}^{-2}$, while in Fields 1 and 2 combined it is $3.2 \pm 0.4 \operatorname{arcmin}^{-2}$. Assuming that the objects near the red sequence in Figure 3 are binaries, then the frequency of unresolved equal mass binaries in the Center and Shoulder regions is $0.6 \pm 0.1$. This is comparable to the frequency of equal mass binaries among MS stars in the open cluster NGC 3105, which Davidge (2017) finds is at least 10 Myr older than Haffner 16.

\subsection{Comparisons with Isochrones}

The combined CMD of the Center and Shoulder regions is compared with $\mathrm{Z}=0.016$ PARSEC (Bressan et al. 2012) isochrones in Figures 5 and 6 . The models were downloaded from the Padova database of stellar evolutionary tracks and isochrones website 4 . The composite CMD of Fields 1 and 2 is also shown for comparison. Isochrones with other metallicities are considered later in this section.

Davidge et al. (2013) considered distance moduli

\footnotetext{
${ }^{4}$ http://pleiadi.pd.astro.it/
} 


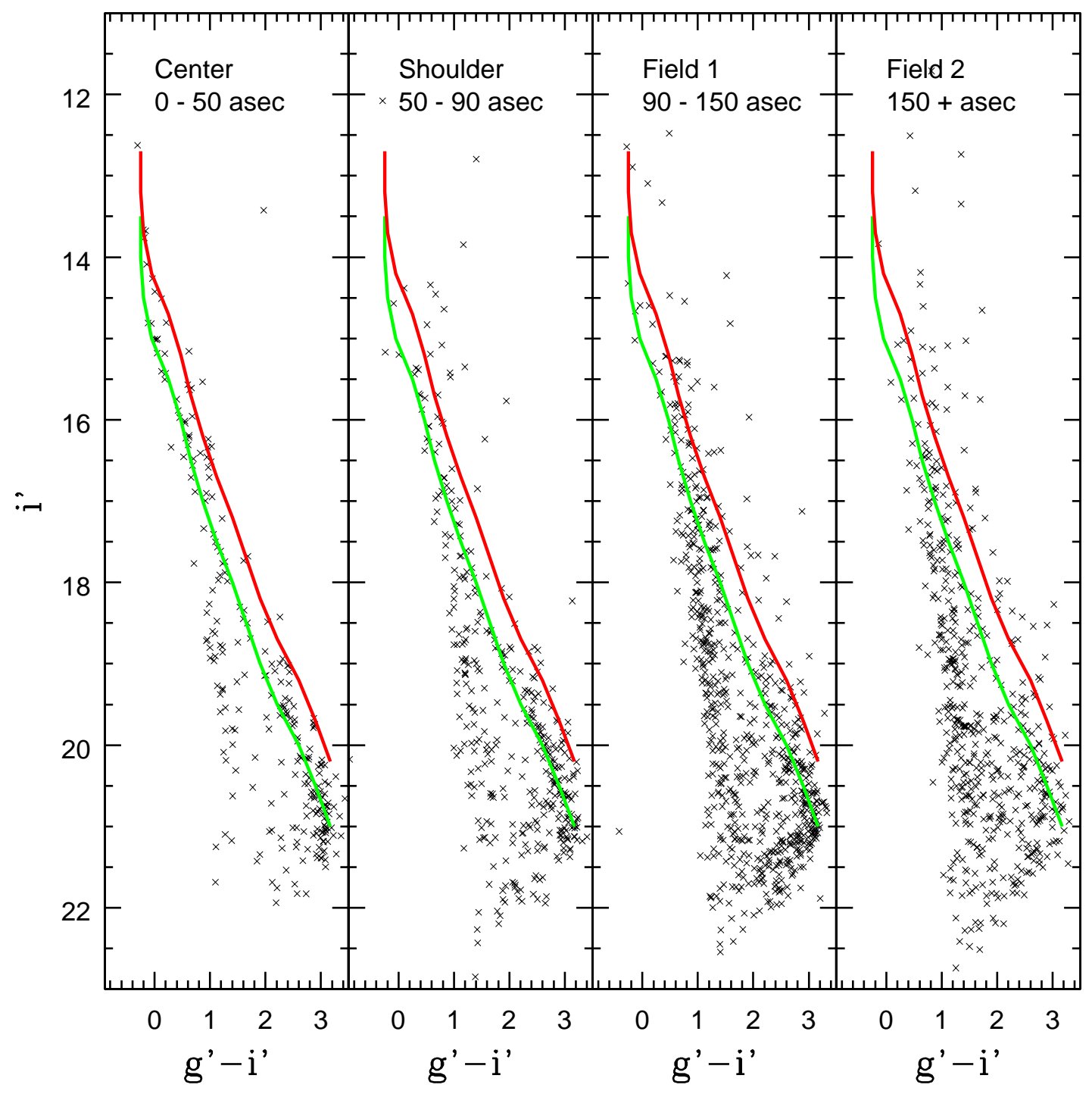

FIG. 3.- $\left(i^{\prime}, g^{\prime}-i^{\prime}\right)$ CMDs of stars in and around Haffner 16. The locus of upper MS stars in Haffner 16 is seen in the Center region CMD at $i^{\prime}<16$, while a well-defined PMS slices diagonally across the Center and Shoulder CMD when $i^{\prime}>18$. A steep blue sequence that is made up of disk stars is seen in the Field 1 and 2 CMDs. The green line is a hand drawn representation of the cluster sequence in the Center and Shoulder CMDs. The red line is this same fiducial, but shifted upwards by 0.75 magnitudes to mark the expected location of unresolved equal mass binaries in Haffner 16. There are sources in the Center and Shoulder CMDs that fall close to the binary sequence, and in the text it is shown that these have markedly higher densities on the sky than objects with similar photometric properties in Fields 1 and 2 .

of 12.5 and 13.5 for Haffner 16 , and the results of adopting these are explored in Figures 5 and 6. The isochrones have been positioned to match the blue envelope of the brightest MS stars in the left hand panel of each figure, and this sets the reddening. A distance modulus of 12.5 is adopted for Figure 5. The three older isochrones match the inflexion point in the cluster CMD near $i^{\prime}=15$. The 4 and 10 Myr isochrones fall well above the cluster sequence over much of the CMD, while the 32 and $71 \mathrm{Myr}$ isochrones fall below the cluster sequence near the faint end of the CMD. The cluster sequence is best matched by the 20 Myr isochrone at magnitudes $i^{\prime} \leq 18$. However, at fainter magnitudes the 20 Myr model falls progressively to the left of the PMS. There is a $\sim 0.5$ magnitude difference in $g^{\prime}-i^{\prime}$ between the $20 \mathrm{Myr}$ isochrone and the cluster sequence near $i^{\prime}=21$.
A distance modulus of 13.5 is adopted for Figure 6 . The agreement between the cluster locus and the isochrones is much poorer than in Figure 5, and none of the isochrones in this figure match the inflexion point in the cluster sequence near $i^{\prime}=15$. The isochrones with ages $\geq 20$ Myr pass through the blue plume of objects when $\bar{i}^{\prime}>18$, which are shown to be non-cluster stars in Figure 4 .

The comparisons in Figures 5 and 6 indicate that a distance modulus close to 12.5 is favored if Haffner 16 has a metallicity that is near solar. It is also apparent that models with a range of ages can match the MS of Haffner 16 at magnitudes $i^{\prime}<16$. Subtle structure in the CMD that might provide additional insights into age and distance is hard to distinguish when the full magnitude range of the CMD is shown as in Figures 5 and 6 . 


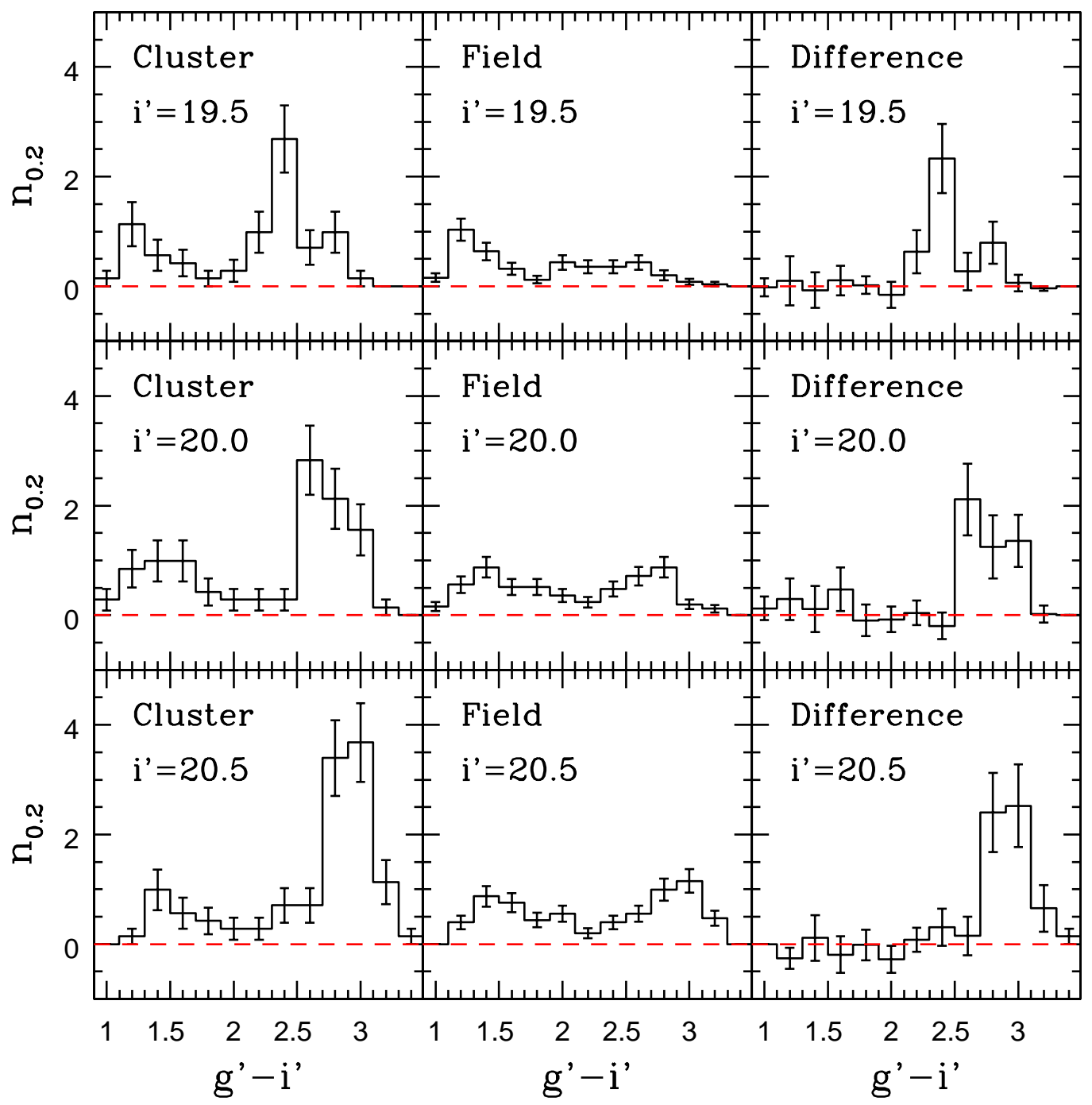

FIG. 4. $-g^{\prime}-i^{\prime}$ color distributions near the faint end of the CMDs. $\mathrm{n}_{0.2}$ is the number of stars $\operatorname{arcmin}^{-2}$ per 0.2 magnitude interval in $g^{\prime}-i^{\prime}$ with \pm 0.25 magnitude binning in $i^{\prime}$. The error bars are $1 \sigma$ uncertainties computed with Poisson statistics (i.e. the uncertainty in each bin is the square root of the number of counts, with errors in the background counts propagated in quadrature). The dashed red line in each panel marks $\mathrm{n}_{0.2}=0$. Color distributions are shown for the Cluster+Shoulder region (left hand column), Field $1+2($ middle column), and the difference between the two (right hand column). To the extent that Fields 1 and 2 monitor non-cluster members then the right hand panel shows the $g^{\prime}-i^{\prime}$ distribution of stars in Haffner 16. Note that objects with $g^{\prime}-i^{\prime}<2.0$ in each magnitude interval subtract out, indicating a uniform distribution on the sky - these objects are therefore not cluster members.

Therefore, the CMD of Haffner 16 in a narrower range of magnitudes - that includes the likely location of the MSCO - is shown in Figure 7.

Haffner 16 might be expected to have a slightly lower than solar metallicity, as there is evidence of a metallicity gradient with a slope $\sim-0.04$ dex $\mathrm{kpc}^{-1}$ among young objects in the Galactic disk (e.g. Daflon \& Cunha 2004; Balser et al. 2011). If Haffner 16 falls along this trend then it would have a metallicity that is $\sim 0.1$ dex lower than solar, and the spectroscopic properties of stars in and around Haffner 16 appear to favor a sub-solar metallicity (Section 7 ). There is $\sim \pm 0.2$ dex scatter about the mean metallicity trends in the Daflon \& Cunha (2004) and Balser et al. (2011) studies. Adopting $\mathrm{Z}=0.016$ as solar, then an upper limit for the metallicity of Haffner 16 is $\mathrm{Z}=0.020$, while a lower limit is
$\mathrm{Z}=0.008$. Comparisons are made with models that have these metallicities in Figure 7.

The distance modulus for each isochrone has been adjusted to give the best 'by eye' match to the cluster sequence, and the distance moduli are listed in both panels. The distance moduli found with the $\mathrm{Z}=0.008$ isochrones are 0.4 dex smaller than those estimated from the $\mathrm{Z}=0.020$ models. The two sets of models yield a 0.02 magnitude difference in $\mathrm{E}(\mathrm{B}-\mathrm{V})$.

The cluster fiducial is not well-defined near $i^{\prime}=$ 16 , and this illustrates the difficulty estimating to within a few Myr the ages of moderate mass clusters at low Galactic latitudes from CMDs alone. For example, the comparisons in Figure 7 indicate that if Haffner 16 were as young as $10 \mathrm{Myr}$ then there would be a kink in the CMD between $i^{\prime}=15$ and 15.5. Small number statistics 


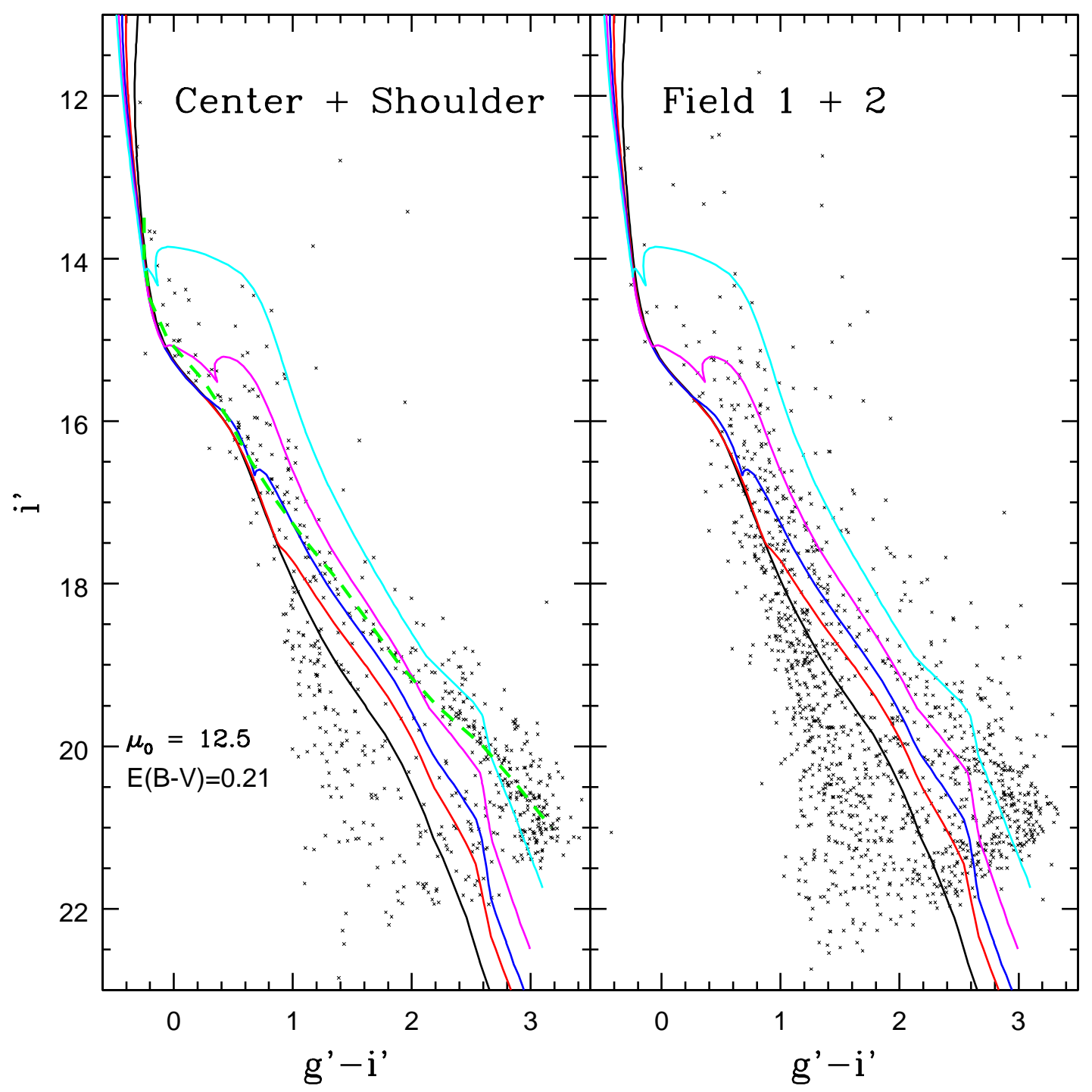

FIG. 5.- Combined CMDs of the Center and Shoulder regions (left hand panel) and Fields 1 and 2 (right hand panel) are compared with $\mathrm{Z}=0.016$ isochrones from Bressan et al. (2012). A distance modulus of 12.5 is assumed. $\mathrm{E}(\mathrm{B}-\mathrm{V})=0.21$ is found by matching the isochrones to the blue edge of the bright $\mathrm{MS}$ in the left hand panel. $\mathrm{E}(\mathrm{B}-\mathrm{V})$ was computed from $\mathrm{E}(\mathrm{g}$ ' $-\mathrm{i}$ ') using the reddening relations in Table 6 of Schlegel et al. (1998). Isochrones with ages 4 Myr (cyan), 10 Myr (magenta), 20 Myr (dark blue), 32 Myr (red), and 71 Myr (black) are shown. The dashed green line in the left hand panel is the cluster locus from Figure 3 . The 4 and 10 Myr models do not match the inflexion point in the cluster sequence near $i^{\prime}=15$, but come close to matching the colors of PMS stars at the faint end. While the 20 Myr model matches the cluster sequence when $i^{\prime}>18$, it falls $\sim 0.5$ magnitudes to the left of the cluster PMS near $i^{\prime}=21$.

make the detection of such a feature in Haffner 16 problematic. Still, the $10 \mathrm{Myr}$ isochrone lies $\sim 0.3$ magnitudes in $g^{\prime}-i^{\prime}$ redward of the fiducial between $i^{\prime}=15.5$ and 17 . While there are points with $g^{\prime}-i^{\prime} \sim 0.6$ and $i^{\prime} \sim 15.6$ that fall along the $10 \mathrm{Myr}$ isochrone, there is not a significant excess density of points in this part of the CMD when compared with densities measured in Fields 1 and 2. The stars that fall along the $10 \mathrm{Myr}$ isochrone near $i^{\prime}=15.6$ also have spectroscopic characteristics that are consistent with them being field stars (Section 7). In contrast, the stars with bluer colors that fall near the cluster fiducial have spectroscopic characteristics that are in line with them being cluster MS stars (Section 7).

A better match between the $10 \mathrm{Myr}$ isochrone and the cluster sequence near $i^{\prime}=15$ could be obtained by adopting a distance modulus of 12.5 - 12.6, but then there are difficulties matching the cluster locus at fainter magnitudes. In Section 6 it is shown that the $i^{\prime}$ LF of Haffner 16 does not match models with an age of 10 Myr unless a distance modulus of 13.8 is adopted, and this is clearly not consistent with the CMD in Figure 7. Therefore, the photometric data indicate an age in excess of 10 Myr for Haffner 16.

The 14 Myr sequence is a better match to the observations than the 10 Myr model at magnitudes $i^{\prime}<16$. Still, the 14 Myr isochrone falls to the red of the fiducial at fainter magnitudes. The $14 \mathrm{Myr}$ model has a kink in the CMD near $i^{\prime}=16$, although the identification of this feature in the CMD would likely require more stars than are present in Figure 7. Of the three models shown in Figure 7, the 20 Myr sequence gives the best overall match to the cluster sequence, although the reader is 


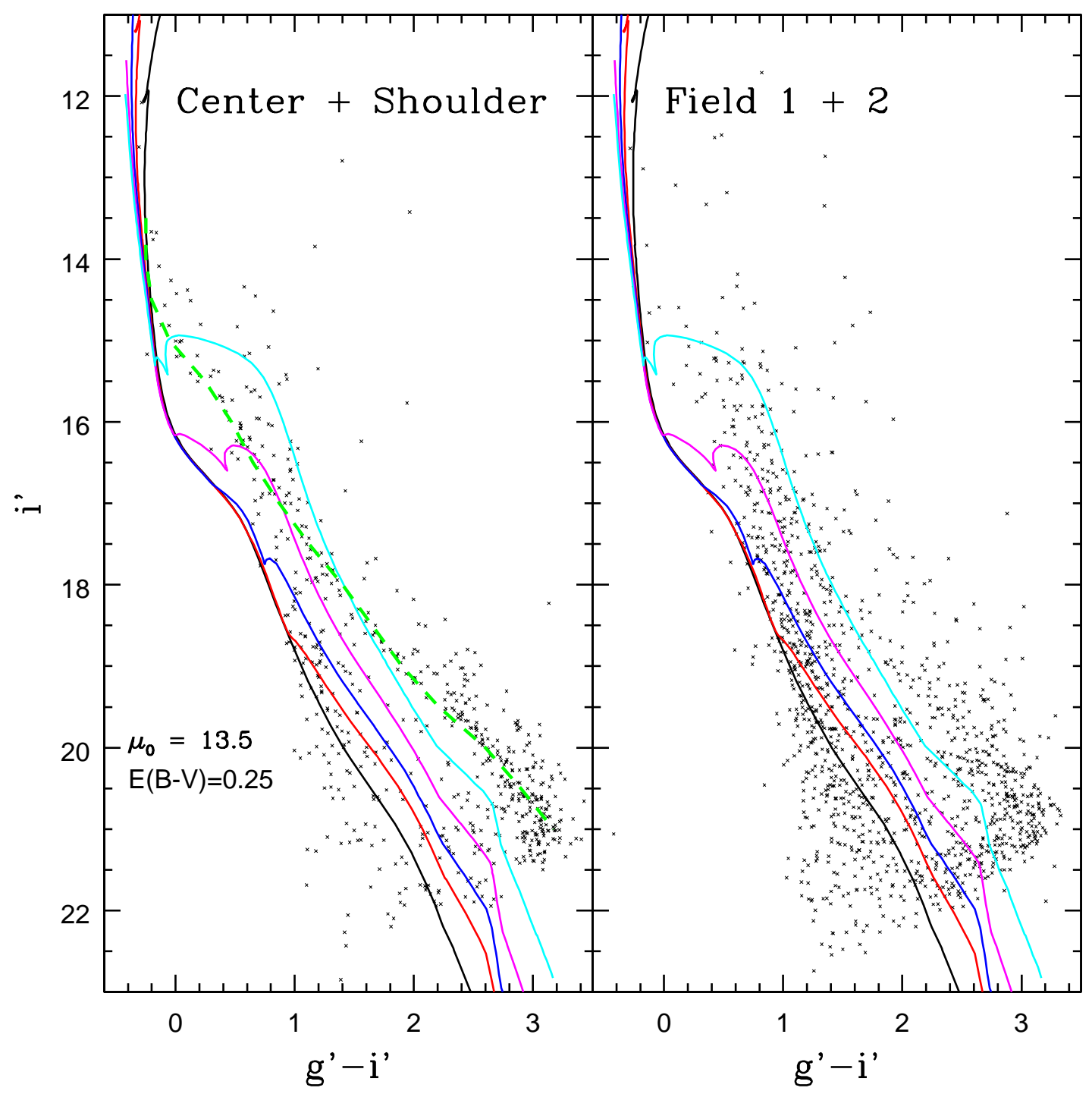

FIG. 6. - Same as Figure 5, but with a distance modulus of 13.5 and $\mathrm{E}(\mathrm{B}-\mathrm{V})=0.25$. The models with ages $\geq 10$ Myr do not reproduce the inflexion point in the CMD near $i^{\prime}=15$, and all models fall to the left of the PMS at the faint end of the $\overline{C M D}$.

again reminded that there is uncertainty in the cluster locus at the magnitudes shown in the Figure. In Section 6 it is shown that models with an age of $20 \mathrm{Myr}$ also match the $i^{\prime}$ LF of Haffner 16. Therefore, based on the results in Figure 7 we assign Haffner 16 a distance modulus of $12.3 \pm 0.2$.

\section{THE CLUSTER LUMINOSITY FUNCTION}

The $i^{\prime}$ LFs of the Center, Shoulder, and combined Center+Shoulder regions are shown in Figure 8. Only objects detected in both $g^{\prime}$ and $i^{\prime}$ have been counted. A correction for non-cluster objects was applied by subtracting stellar densities measured in Fields 1 and 2. As some cluster stars may be present in Fields 1 and 2, this may result in the over-subtraction of stars, which could affect the slope of the LF. However, as the Center and Shoulder regions were selected in Section 4 on the basis of having a clear over-density of objects with respect to the surroundings then over-subtraction is not expected to have a significant effect on the LFs.
There is a break in counts between $i^{\prime}=16.5$ and 17.0 in the Center field LF. Although less significant statistically, there is also evidence for a similar change in character in the LF of the Shoulder region at the same magnitude. The $K$ LF of Haffner 16 constructed by Davidge et al. (2013) has a similar break, in this case near $K=16$. The Davidge et al. (2013) LF was constructed from a smaller science field, and used counts from the Robin et al. (2003) model Galaxy to correct for contamination by non-members. That a change in the character of the LF is seen at different wavelengths and fields of view indicates that the feature near $i^{\prime}=17$ in Figure 8 is not due to a fluke over-density of objects in Fields 1 and 2. That this feature is seen in both the Center and Shoulder regions also indicates that it is not due to a stochastic under-density of objects in one region. Rather, the change in the LF near $i^{\prime}=17$ (and near $K=16$ in the Davidge et al. LF) probably reflects an intrinsic property of Haffner 16 .

The MSCO can produce a prominent feature in 


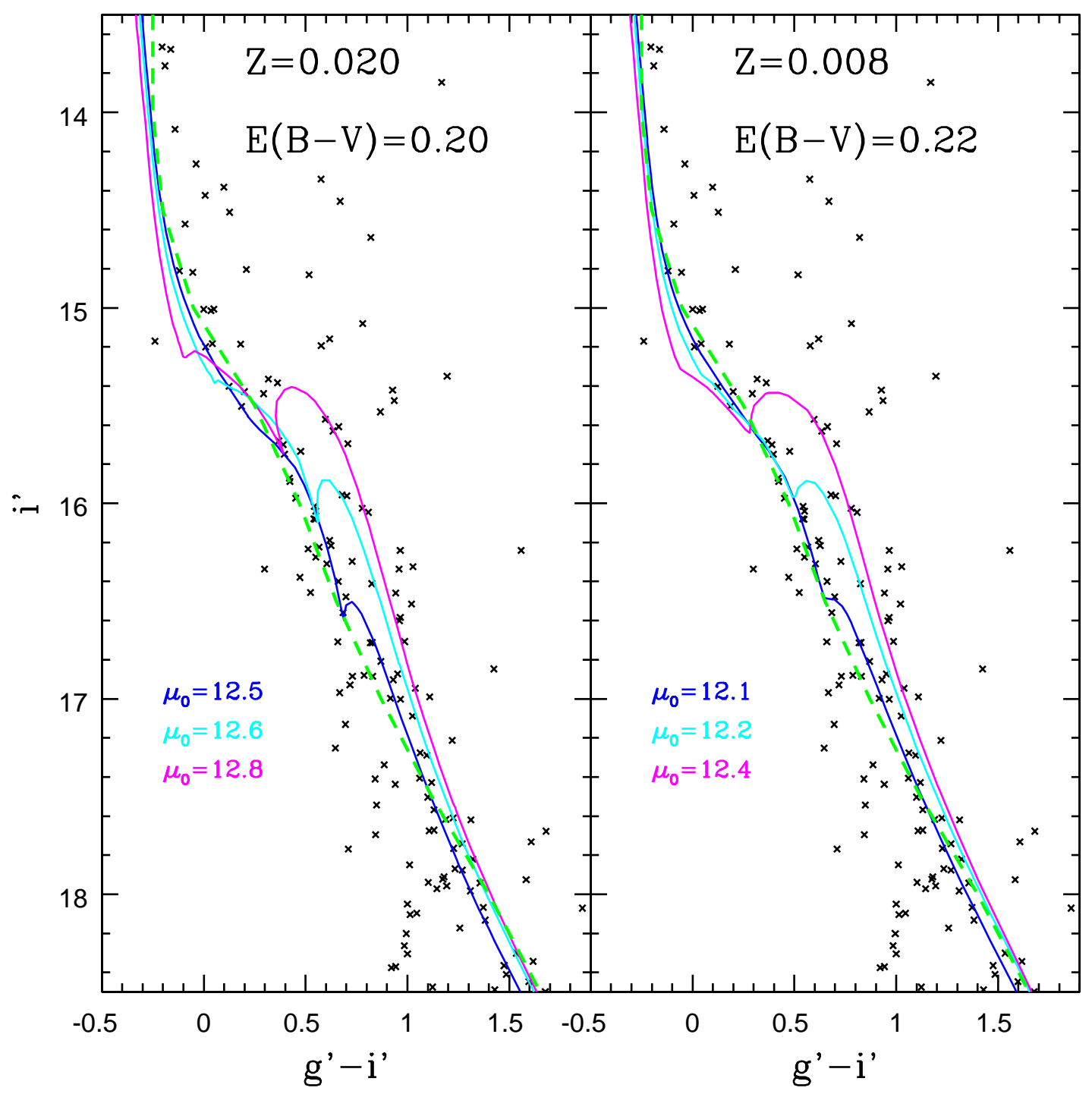

FIG. 7.- Comparing the combined CMD of the Cluster and Shoulder regions with $\mathrm{Z}=0.020$ (left hand panel) and $\mathrm{Z}=0.008$ (right hand panel) isochrones near the expected brightness of the MSCO. These metallicities span the range seen among objects at the Galactocentric radius of Haffner 16. Isochrones from Bressan et al. (2012) with ages of 10 (magenta), 14 (cyan), and 20 (blue) Myr are shown. The dashed green line is the cluster locus from Figure 3. The distance modulus of each isochrone has been adjusted to match the cluster locus. The distance moduli found from the $\mathrm{Z}=0.008$ models are 0.4 dex lower than those from the $\mathrm{Z}=0.020$ models.

LFs (e.g. Cignoni et al. 2010), and the break in the Haffner $16 \mathrm{LF}$ near $i^{\prime}=17$ is likely due to the MSCO. This is demonstrated in Figure 8, where model LFs constructed from $\mathrm{Z}=0.012$ (i.e. $[\mathrm{M} / \mathrm{H}] \sim-0.2$ ) Padova isochrones are shown. As discussed in Section $5.1, \mathrm{Z}=0.012$ is the metallicity expected for Haffner 16 if the cluster follows the relations between metallicity and Galactocentric distance found by Daflon \& Cunha (2004) and Balser et al. (2011). Based on the comparisons in Figure 7, a distance modulus of 12.6 is assumed for the 10 Myr model, while a distance modulus of 12.3 is assumed for the $20 \mathrm{Myr}$ model. The models in Figure 8 assume a system that is populated by single stars (i.e. no binaries). A Chabrier (2003) mass function has been adopted.

Model LFs were also generated for $\mathrm{Z}=0.020$ (i.e. $[\mathrm{M} / \mathrm{H}] \sim+0.1)$. While not shown here, these models are similar in appearance to the $\mathrm{Z}=0.012 \mathrm{LFs}$, and provide similar agreement with the Haffner 16 LF after adopting distances based on the comparisons between isochrones and CMDs (Section 5). This similarity in LF shape indicates that the comparisons in Figure 8 are not sensitive to metallicity.

The MSCO produces a large discontinuity near $i^{\prime}=16$ in the 10 Myr model with an amplitude that is similar to the dip in the Haffner 16 LF. However, the MSCO in the 10 Myr model occurs 1 magnitude brighter than this feature in the Haffner $16 \mathrm{LF}$, and does not match the counts near $i^{\prime}=16$ and 16.5 in the bottom panel of Figure 8. While a distance modulus of 13.6 would produce much better agreement between the 10 Myr model and the LF, such a distance modulus is not consistent with the morphology of the Haffner 16 CMD, as demonstrated in Figures 6 and 7.

The MSCO produces a peak in the 20 Myr model LF near $i^{\prime}=16.5$. The 20 Myr model matches the 


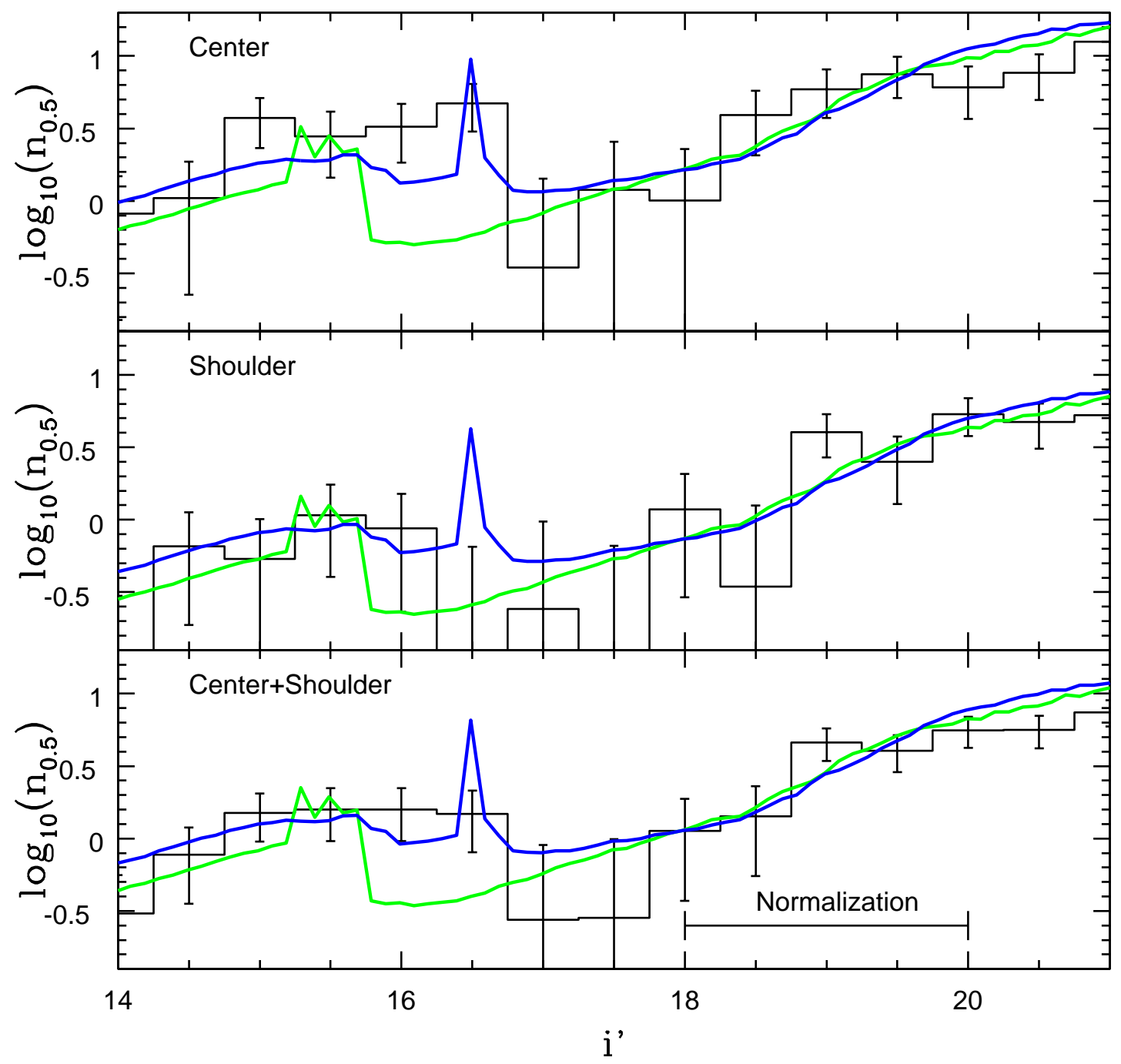

FIG. 8. - $i^{\prime}$ LFs of the Center, Shoulder, and combined Center+Shoulder regions. $\mathrm{n}_{0.5}$ is the number of objects arcmin ${ }^{-2}$ in each 0.5 magnitude interval. The LFs have been corrected for non-cluster sources by subtracting number counts from Fields 1 and 2 , scaled to match the areal coverage of the Center and Shoulder regions. The error bars are $1 \sigma$ uncertainties calculated from Poisson statistics. The green and blue lines are model LFs for single stars (i.e. no binaries) constructed from $\mathrm{Z}=0.012$ PARSEC isochrones with ages of 10 and $20 \mathrm{Myr}$. The models assume $\mathrm{E}(\mathrm{B}-\mathrm{V})=0.21$ and distance moduli of 12.6 (10 Myr) and $12.3(20 \mathrm{Myr})$. The models have been normalized to match the observed LFs between $i^{\prime}=18$ and 20 . The 20 Myr model matches the Center+Shoulder LF within the $\pm 2 \sigma$ level at all brightnesses. In contrast, the $10 \mathrm{Myr}$ model significantly underestimates the counts at $i^{\prime}=16$ and 16.5 .

LF in the bottom panel of Figure 8 at the $\pm 2 \sigma$ level from $i^{\prime}=14$ to $i^{\prime}=20$. That the $20 \mathrm{Myr}$ model agrees with the observations within the error bars leads us to conclude that the LF is consistent with an age $\sim 20$ Myr for Haffner 16. This agreement also indicates that the Chabrier (2003) mass function can replicate number counts in Haffner 16, at least in the mass range probed by these data.

Davidge et al. (2013) found evidence for mass segregation in Haffner 16, in the sense that the brightest MS stars were more centrally concentrated than the fainter, lower mass stars in the $K$ LF. However, the LFs of the Center and Shoulder regions in Figure 8 are not significantly different, and so there is no evidence of mass segregation. The GMOS observations sample a larger area than was observed by Davidge et al. (2013) $(5.5 \times 5.5$ arcmin vs. $1.5 \times 1.5$ arcmin). This allows field star contamination to be monitored empirically with the GMOS data, whereas Davidge et al. (2013) relied on model star counts. Such models may be uncertain in individual low Galactic fields due to - for example - localized non-uniformities in the line-of-sight extinction.

In Section 5.1 it was shown that a significant fraction of stars in Haffner 16 might be in equal mass binaries. Figure 9 shows the Haffner 16 LFs compared with models that assume a mix of single stars and equal mass binaries, with a binary fraction of 0.6. The models were constructed by combining two single star LFs, one of which was shifted to brighter values by 0.75 magnitudes to simulate the LF of equal mass binaries. The models in Figure 9 thus differ from the single star models in that there are two MSCO bumps - the fainter of these is due 
to single stars, while the brighter originates in equal mass binary systems. These models assume an idealized case in which the component stars have not interacted, and are evolving in lockstep.

The 10 Myr models that include binaries are an inferior representation of the observations when compared with the single star models in Figure 8 . The same is true for the 20 Myr models between $i^{\prime}=15$ and 16, although at all brightnesses the $20 \mathrm{Myr}$ model in Figure 9 matches the observations within the $2 \sigma$ uncertainties. The binary fraction was measured in a relatively narrow range of magnitudes that sampled only the PMS (Section 5.1), and it is possible that it may not hold over a broader range of magnitudes.

\section{STELLAR SPECTRA}

\subsection{General Properties of the Spectroscopic Sample}

The $\left(i^{\prime}, g^{\prime}-i^{\prime}\right)$ CMD of the stars targeted for spectroscopy is shown in Figure 10. The restricted magnitude range of the targets in each mask is evident. The four stars that are in isolated parts of the CMD were observed only because they could be placed in the same slit as an object that was a primary target for spectroscopy. The spectroscopic properties of these objects are discussed in Section 7.4.

Haffner 16 is a young cluster, and so it is not surprising that some of its members are emission line sources. The location on the CMD of stars that have $\mathrm{H} \alpha$ either in absorption (black squares) or emission (magenta squares) are indicated in Figure 11. None of the stars with $i^{\prime}<18$ show obvious $\mathrm{H} \alpha$ emission, in agreement with the low incidence of emission found among early-type stars in this part of the sky by McSwain \& Gies (2005). Weak line emission might be hard to detect in the deep $\mathrm{H} \alpha$ profiles of the stars at brighter magnitudes.

Figure 12 shows the locations of the spectroscopic targets in the GMOS science field, with stars marked according to whether or not they have $\mathrm{H} \alpha$ in emission. The stars that have $\mathrm{H} \alpha$ in absorption are scattered moreor-less uniformly across the field, whereas the emission line sources tend to be concentrated in the Center and Shoulder regions. This is due in part to the (intentional) bias to obtain spectra of faint stars in the main body of the cluster, where the incidence of cluster members is highest. Still, some emission line sources are seen outside of the Shoulder region.

The stars selected for spectroscopic follow-up have a range of photometric properties, and were sorted into seven groups according to location on the CMD. The group boundaries are indicated in Figure 10. Groups 1, $3,5,6$, and 7 are made up of objects on or near the cluster sequence. Groups 5, 6, and 7 overlap on the CMD, and the behaviour of the $\mathrm{H} \alpha$ line is used to distinguish between members of these three groups: stars in group 5 have weak $\mathrm{H} \alpha$ absorption, while stars in groups 6 and 7 have either weak (group 6) or strong (group 7) $\mathrm{H} \alpha$ emission.

The members of each group are listed in Table 3. The identification number for each target consists of the mask \# (1-6), followed by the slit number, defined such that slitlet \# 1 for each mask is the closest to the bottom of the science field. The 'a' and 'b' designations indicate
TABLE 3

Group MEMBERShIP

\begin{tabular}{|c|c|}
\hline Group & Star IDs \\
\hline 1 & $\begin{array}{l}(1-01),(1-02), 1-03,1-04,(1-05) \\
1-06,1-07,1-08,(1-09),(1-10) \\
(1-11), 1-12,1-14,(2-01),(2-02) \\
2-03,(2-05), 2-06,2-07,(2-09) \\
(2-10),(2-11)\end{array}$ \\
\hline 2 & $\begin{array}{l}(1-13),(1-15),(1-16), 2-04,2-08 \\
2-12,(2-13),(2-15),(2-16)\end{array}$ \\
\hline 3 & $\begin{array}{l}3-05 a,(3-07),(3-09),(3-16),(3-17) \\
3-21,(3-22), 4-03,(4-07),(4-09) \\
4-12,4-14 a, 4-14 b,(4-17),(4-18) \\
4-19,4-21 a,(4-23),(5-15),(6-07)\end{array}$ \\
\hline 4 & $\begin{array}{l}(3-01), 3-02,3-03,(3-04),(3-06) \\
3-08,3-10,3-11,3-12,3-13 \\
3-14,3-15,3-18,(3-19), 3-20 \\
(4-01), 4-02,(4-04), 4-05,(4-06) \\
4-08,4-10,4-11 \mathrm{a}, 4-11 \mathrm{~b}, 4-13 \mathrm{a} \\
4-13 \mathrm{~b}, 4-15,4-16,(4-20),(4-22) \\
(5-01),(5-19),(6-01),(6-10)\end{array}$ \\
\hline 5 & $\begin{array}{l}5-02,5-04,5-08,5-14 a, 5-16 \\
5-17,5-18,5-20,5-21,6-02 b \\
6-03\end{array}$ \\
\hline 6 & $\begin{array}{l}5-03,5-05,5-09 a, 5-10,5-11 \\
5-12,5-13,5-14 b, 6-06\end{array}$ \\
\hline 7 & $\begin{array}{l}5-06,5-07,6-04 a, 6-04 b, 6-05 \\
6-08,6-09\end{array}$ \\
\hline
\end{tabular}

stars that are in the same slitlet. The limited number of stars with $i^{\prime}<16$ causes significant overlap in the object lists for Masks $1+2$, and $3+4$. The co-ordinates and photometric properties of stars with spectra are given in Table 4. Stars that have multiple observations are indicated with an asterix, with their identification numbers in other masks given in brackets.

A median spectrum was constructed for each group, and the results in the 5300 to $6800 \AA$ interval, which is the wavelength interval that is common to most stars, are shown in Figure 13. The spectra in this figure have been convolved with a Gaussian to produce a resolution of 640 to improve the $\mathrm{S} / \mathrm{N}$ ratio of the spectra of stars in groups 5,6 , and 7 . This spectral resolution is sufficient to allow absorption features that are diagnostics of basic stellar properties to be detected (e.g. Worthy et al. 1994). Aside from $\mathrm{Na} \mathrm{D}$ and $\mathrm{H} \alpha$, many of the absorption features at these wavelengths are Fe I and Ca I transitions, although other atomic and molecular species contribute (e.g. Wallace et al. 2011). Applying Equation 9 of Poznanski et al. (2012) suggests that interstellar absorption towards Haffner 16 likely contributes an equivalent width of $\sim 1 \AA$ to the $\mathrm{Na} \mathrm{D}$ lines.

Some stars in groups $1-4$ were observed in more than one mask, and so the $\mathrm{S} / \mathrm{N}$ ratio of the processed spectra can be estimated in a statistical manner by comparing the depths of features in multiple spectra of the same star. Groups $1-4$ sample stars with $i^{\prime}<16$, and the spectra in these groups have comparable $\mathrm{S} / \mathrm{N}$ ratios. Consider the depths of $\mathrm{Na} \mathrm{D}$ and $\mathrm{H} \alpha$, which are the strongest features in the spectra of stars in these groups. The dispersion in the depths of $\mathrm{Na} \mathrm{D}$ among stars with multiple spectra in groups $1-4$ is only $\pm 1 \%$, whereas the dispersion in the depth of $\mathrm{H} \alpha$ is $\pm 2 \%$. The dispersions include contributions from photon noise, the centering of the star in the slit, which can affect line shape, sky subtraction, and the placement of the continuum.

There is a star-to-star dispersion in the spectro- 


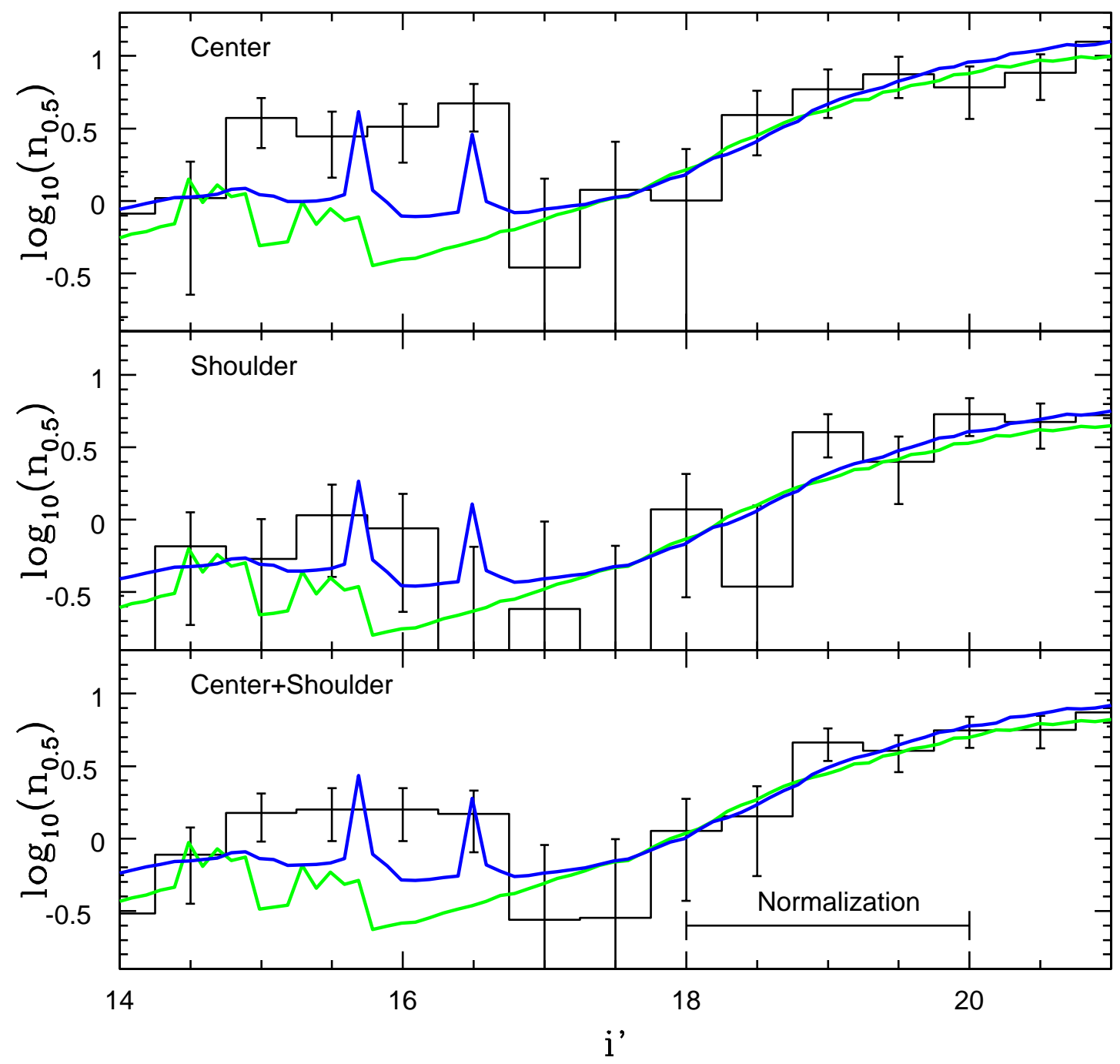

FIG. 9.- Same as Figure 8, but including equal mass binaries. The agreement between the models and observations at the bright end $\left(i^{\prime} \leq 16\right)$ is poorer than in Figure 8.

scopic properties of the members of each group. This scatter is due to slight differences among cluster members in each group (recall that the groups sample stars with a range of magnitude and color) and contamination from non-cluster members. As with the examination of the spectra of stars with repeat measurements, the starto-star scatter in the depths of $\mathrm{Na} \mathrm{D}$ and $\mathrm{H} \alpha$ provides insights into the range of spectroscopic properties in each group. Groups 1 and 3 have the smallest star-to-star scatter, with $\sigma=2 \%$ for $\mathrm{Na} \mathrm{D}$, and $\pm 3 \%$ for $\mathrm{H} \alpha$. These dispersions are only slightly larger than those estimated in the previous paragraph that are due to random and systematic errors in individual spectra. The star-to-star scatter is larger for groups 2 and 4 , with $\sigma= \pm 4 \%$ for $\mathrm{Na} \mathrm{D}$, and $\pm 6 \%$ for $\mathrm{H} \alpha$. That groups 2 and 4 sample a more diverse range of spectroscopic properties is not unexpected, as these groups are made up of stars that are offset from the cluster locus on the CMD, and so are likely members of the field population.

The star-to-star dispersions in the depths of $\mathrm{Na}$
$\mathrm{D}$ and $\mathrm{H} \alpha$ among the spectra of stars in groups 5,6 , and 7 are similar, with $\sigma= \pm 7 \%$ for $\mathrm{Na} \mathrm{D}$, and $\pm 5 \%$ for $\mathrm{H} \alpha$. These groups sample fainter stars than those in groups $1-4$, and the lower $\mathrm{S} / \mathrm{N}$ ratio of the spectra likely contributes to the larger dispersion in the $\mathrm{Na} \mathrm{D}$ depths. Unlike is the case for groups $1-4$, the star-tostar dispersion in $\mathrm{H} \alpha$ is smaller than that for $\mathrm{Na} \mathrm{D}$. This may be due to the membership of groups $5-7$ being based on $\mathrm{H} \alpha$ properties, which has the potential to bias the $\mathrm{H} \alpha$ dispersion measurements within each group.

A cursory examination of the spectra in Figure 13 reveals trends that are broadly consistent with the criteria used to define the groups. The group 1 spectrum has the weakest metallic absorption features and deepest $\mathrm{H} \alpha$ absorption, as expected if the stars in group 1 have earlier spectral-types than those in other groups. $\mathrm{H} \alpha$ absorption in group 3 is weaker than in group 1 and metallic absorption features are more pronounced, which is consistent with the stars in group 3 being intrinsically fainter than those in group 1. 


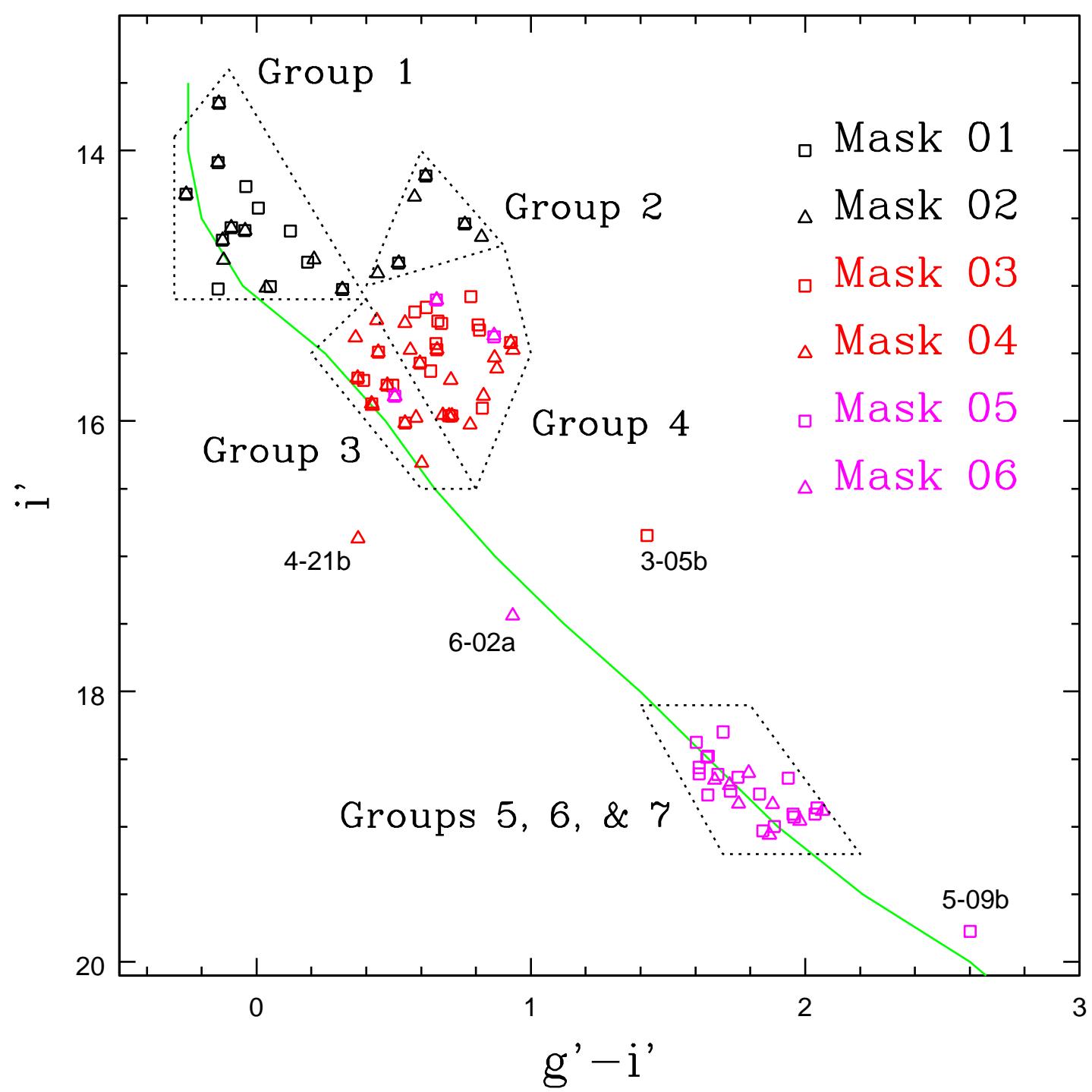

FIG. 10.- CMD of stars with spectra. The green line is the cluster fiducial from Figure 3. The areas on the CMD that define the seven groups discussed in this paper are indicated. A list of the stars in each group can be found in Table 3. The four objects with photometric properties that make them distinct from any group are labelled with their identification numbers.

In contrast to groups 1 and 3 , the spectra of groups 2 and 4 are very similar. The median spectra of these groups have characteristics that are consistent with typical member stars having later spectral-types than those in groups 1 and 3 , in agreement with their $g^{\prime}-i^{\prime}$ colors. That the median spectra of groups 2 and 4 do not show magnitude-related differences is consistent with the majority of stars in these groups belonging to a field population that samples a range of distances. The projected distribution of objects in groups 2 and 4 on the sky is also such that the majority are located outside of the Shoulder region.

If Haffner 16 had a very young age then some PMS stars might be expected in the parts of the CMD that sample groups 2 and 4 . However, while falling to the right of the cluster sequence in Figure 10, the objects in groups 2 and 4 are likely not dominated by PMS stars in Haffner 16. Even adopting an age of $10 \mathrm{Myr}$ and a distance modulus of 12.6 - which together are favorable for the presence of bright PMS stars in Haffner 16 - then PMS stars in Haffner 16 are expected to have $i^{\prime}>15$ (Figure 5), placing any such objects in group 4, and not in group 2. That the median spectra of groups 2 and 4 are similar thus indicates that if PMS stars are present then they are not the dominant population in Group 4.

Aside from differences in the behaviour of $\mathrm{H} \alpha$ that was specified to define group membership, the spectra of Groups 5, 6, and 7 are similar. The characteristic features of these spectra are indicative of a much later spectral-type than stars in the other four groups. The spectroscopic properties of the median spectra of the stars in these - and the other - groups are discussed in greater detail below.

\subsection{Stars with Ho in Absorption}

Composite spectra in the wavelength interval 5300 - $6800 \AA$ of the groups that have $\mathrm{H} \alpha$ in absorption are shown in Figures 14 (groups 1 and 3), 15 (groups 2 and 


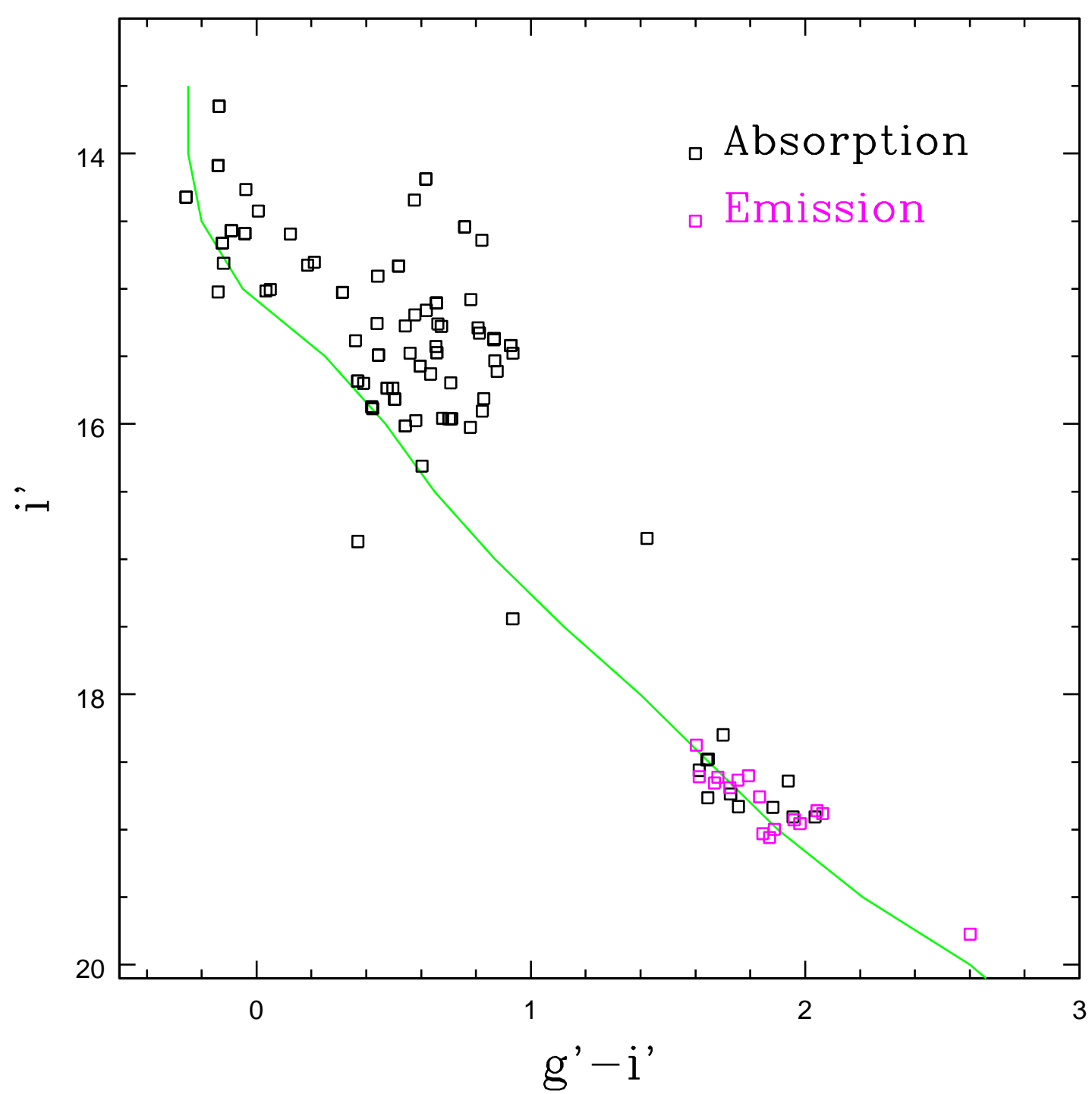

FIG. 11. - CMD of stars with $\mathrm{H} \alpha$ in absorption (black squares) and emission (magenta squares). H $\alpha$ emission is only detected in stars that have $i^{\prime}>18$.

4 ), and 16 (group 5). Spectra from the Le Bourgne et al. (2003) library, smoothed to the same resolution as the Haffner 16 spectra and normalized to the continuum, are also shown. The majority of the stars in the Le Bourgne et al. library are bright and nearby. Thus, as a group they are expected to have roughly solar metallicities.

With the exception of $\mathrm{Na} \mathrm{D}$, which has a contribution from interstellar absorption, the depths of features between 5300 and $6500 \AA$ in the group 1 spectrum in Figure 14 are similar to those in the A1V spectrum. The overall appearance of the group 3 spectrum between 5300 and $6500 \AA$ is consistent with a mid A spectral-type. To quantify these comparisons, various reference stars were subtracted from the group 1 and 3 spectra, and the residuals examined. The $5400-5800 \AA$ and 6000 - $6400 \AA$ wavelength intervals contain numerous metallic features, and the residuals at these wavelengths suggest that the characteristic spectral-type of the group 1 spectrum is early to mid-A. The characteristic spectral-type for group 3 is $\mathrm{A} 5 \mathrm{~V}$. However, the depth of $\mathrm{H} \alpha$ in the group 3 spectrum is more appropriate for an $\mathrm{F}$ spectraltype.

Turning to the spectra of groups 2 and 4 in Figure 15 , the depths of most metallic lines in the $5300-6500 \AA$ interval are consistent with early to mid-F spectral-types. That the group 2 and 4 spectra are similar indicates that the stars in these groups tend to have comparable spectroscopic properties. Subtracting the reference star spectra from the group 2 and 4 spectra and examining the differences in the $5400-5800 \AA$ and $6000-6400 \AA$ intervals suggests that an F5V spectral-type characterizes both groups.

The difference between the mean group 2 and 3 spectra is also shown in Figure 15. With the exception of $\mathrm{Na} \mathrm{D}$ and $\mathrm{H} \alpha$, the residuals scatter about zero. There is a tendency for features to be stronger in the group 2 spectrum when compared with the group 3 spectrum, and this is consistent with the difference in spectral types inferred from the reference stars.

The comparisons in Figure 15 indicate that $\mathrm{H} \alpha$ is 


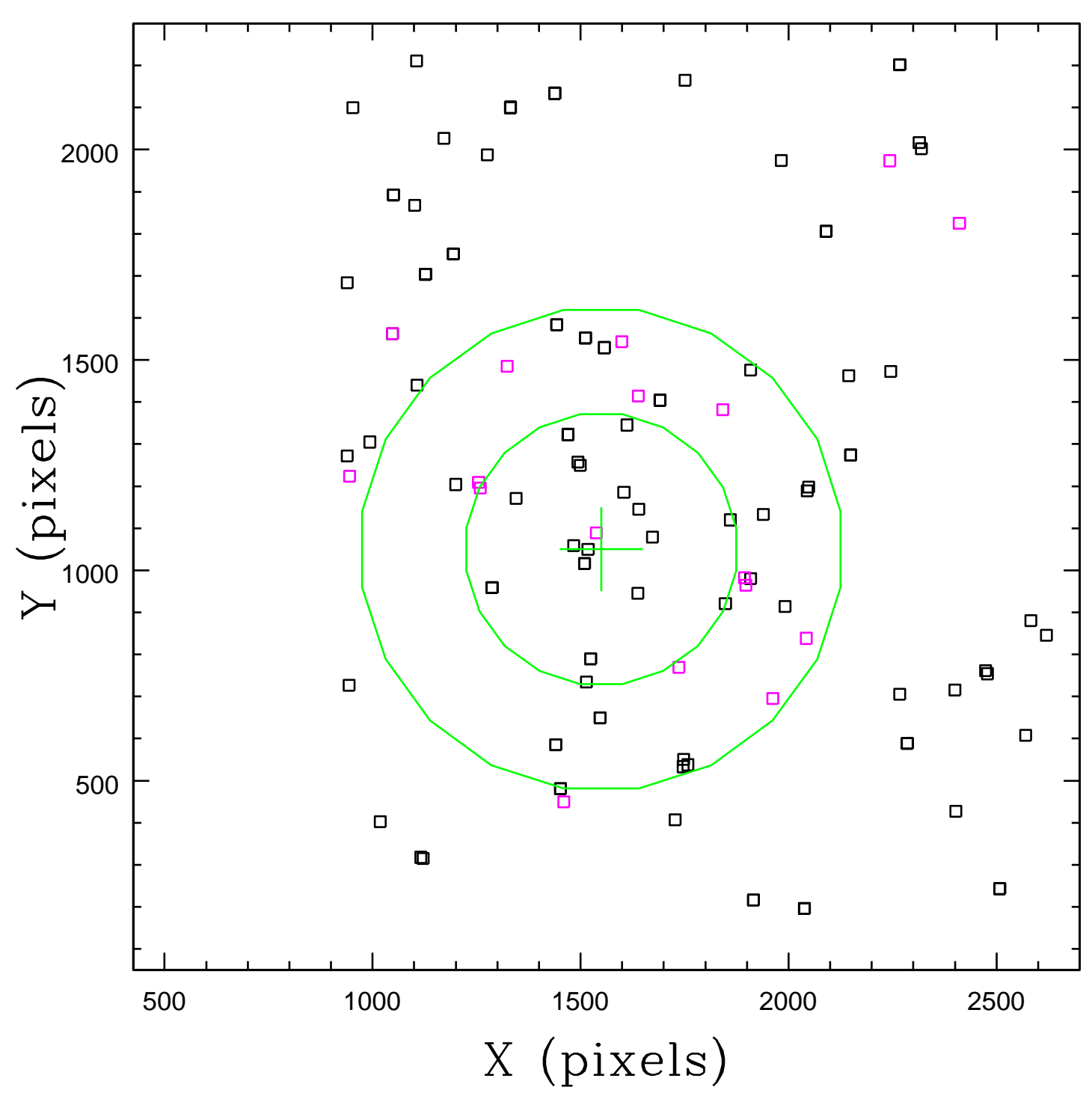

FIG. 12.- Locations of sources that have $\mathrm{H} \alpha$ in absorption (black squares) and emission (magenta squares). $X$ and $Y$ are pixel coordinates on the GMOS detector, and these are listed in Table 3 for each source. The area shown is the same as that displayed in Figure 1. The center of Haffner 16 is marked with a cross, and the circles indicate the boundaries of the Center and Shoulder regions. The majority of emission line sources fall within or just outside of the Shoulder region.

significantly shallower in the group 2 and 4 spectra than is seen among solar neighborhood main sequence stars with mid-F spectral types. What causes $\mathrm{H} \alpha$ to be weaker than expected in the group 2 and 4 spectra? This is likely not a surface gravity effect, as the majority of stars along this line of sight - either in the field or in Haffner $16-$ are expected to be evolving on the main sequence. The similarity of the spectra of groups 2 and 4 also indicates that the relative strengths of metallic lines and $\mathrm{H} \alpha$ are not a fluke arising from a peculiar mix of stars. Indeed, the influence of stars that may have peculiar properties are suppressed by taking the median of spectra in each group. The relative weakness of $\mathrm{H} \alpha$ with respect to the depths of metallic lines when compared with solar neighborhood stars is also likely not due to velocity smearing. Not only do the stars with spectra in this sample have similar velocities, but velocity smearing will affect all absorption features, not just $\mathrm{H} \alpha$. Finally, $\mathrm{H} \alpha$ emission can affect the depth of $\mathrm{H} \alpha$ absorption, while not affecting the depths of metallic lines. However, such emission is not a plausible explanation for the relative weakness of $\mathrm{H} \alpha$ seen here. This is because groups that sample cluster and field members show similar deficiencies in the depth of $\mathrm{H} \alpha$. Remarkably similar levels of $\mathrm{H} \alpha$ emission in the spectra of stars in the cluster and field would then have to be present for this to explain the depths of $\mathrm{H} \alpha$.

The shallow depth of $\mathrm{H} \alpha$ in the spectra of groups 2 and 4 is likely a consequence of a property that is common to the stars along the Haffner 16 line of sight. That Haffner 16 is located outside of the solar circle leads us to suspect that this property is metallicity, in the sense that the majority of stars in our spectroscopic sample have a metallicity that is lower than that of stars that make up the reference spectra. To understand how the depth of $\mathrm{H} \alpha$ can be affected by metallicity, consider two hypothetical stellar systems, one containing metal-poor stars and 


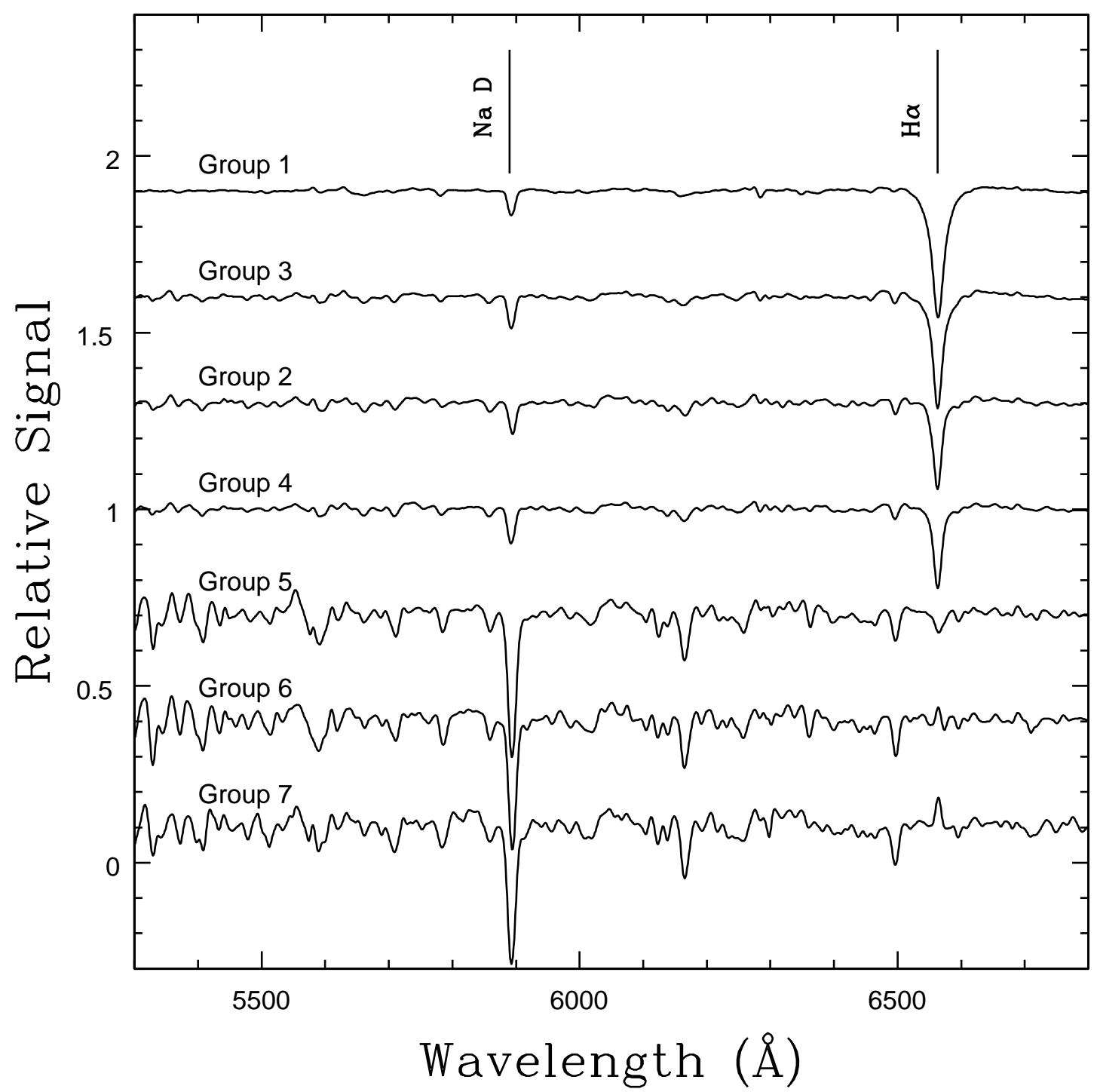

FIG. 13.- Median spectra. The spectra have been shifted vertically for display purposes, and have been smoothed to a resolution of 640 . Note that the spectra are not ordered sequentially by group number.

the other containing metal-rich stars. At a fixed effective temperature the depths of metallic features scale with metallicity - the depths of metallic features in the spectra of stars in the metal-poor system will be shallower than those in the spectra of stars in the metal-rich system that have the same effective temperature. However, the strengths of metallic features at visible wavelengths increase as effective temperature decreases. Spectra of stars in the metal-poor system will have metallic lines with depths that are similar to those seen among stars in the metal-rich system that have higher effective temperatures. The Balmer lines are less susceptible to metallicity variations as the percentage difference in Hydrogen content between the two hypothetical systems is much smaller than that between metals, and so the depths of the Balmer lines are mainly set by effective temperature - the depths of Balmer absorption in stars in the metalrich and metal-poor systems that have similar metal line strengths will then differ. That $\mathrm{H} \alpha$ in the group 3 spectrum is shallower than expected based on the strengths of metallic features when compared with the solar neighborhood thus suggests that Haffner 16 - like the field stars in groups 2 and 4 - has a sub-solar metallicity.

The composite spectrum of stars in group 5 is compared with those of late-type solar neighborhood stars in Figure 16. The majority of metallic features in the Group 5 spectrum have depths that match those in the $\mathrm{K} 0 \mathrm{~V}$ and $\mathrm{K} 2 \mathrm{~V}$ spectra. The absence of $\mathrm{TiO}$ absorption, which is seen in the $\mathrm{K} 7 \mathrm{~V}$ spectrum between 6100 and $6500 \AA$, is consistent with a spectral-type not later than mid-K. Various reference spectra were subtracted from the group 5 spectrum, and the residuals in the $5400-$ $5800 \AA$ and $6000-6400 \AA$ intervals are consistent with a $\mathrm{K} 2 \mathrm{~V}$ characteristic spectral-type for the group 5 spectrum. As was seen amongst the brighter stars, $\mathrm{H} \alpha$ absorption in the group 5 spectrum is weaker than expected based on the depths of metallic features, again hinting at a sub-solar metallicity.

A sub-sample of stars have spectra that extend to $4800 \AA$, and these are objects that are located to the 


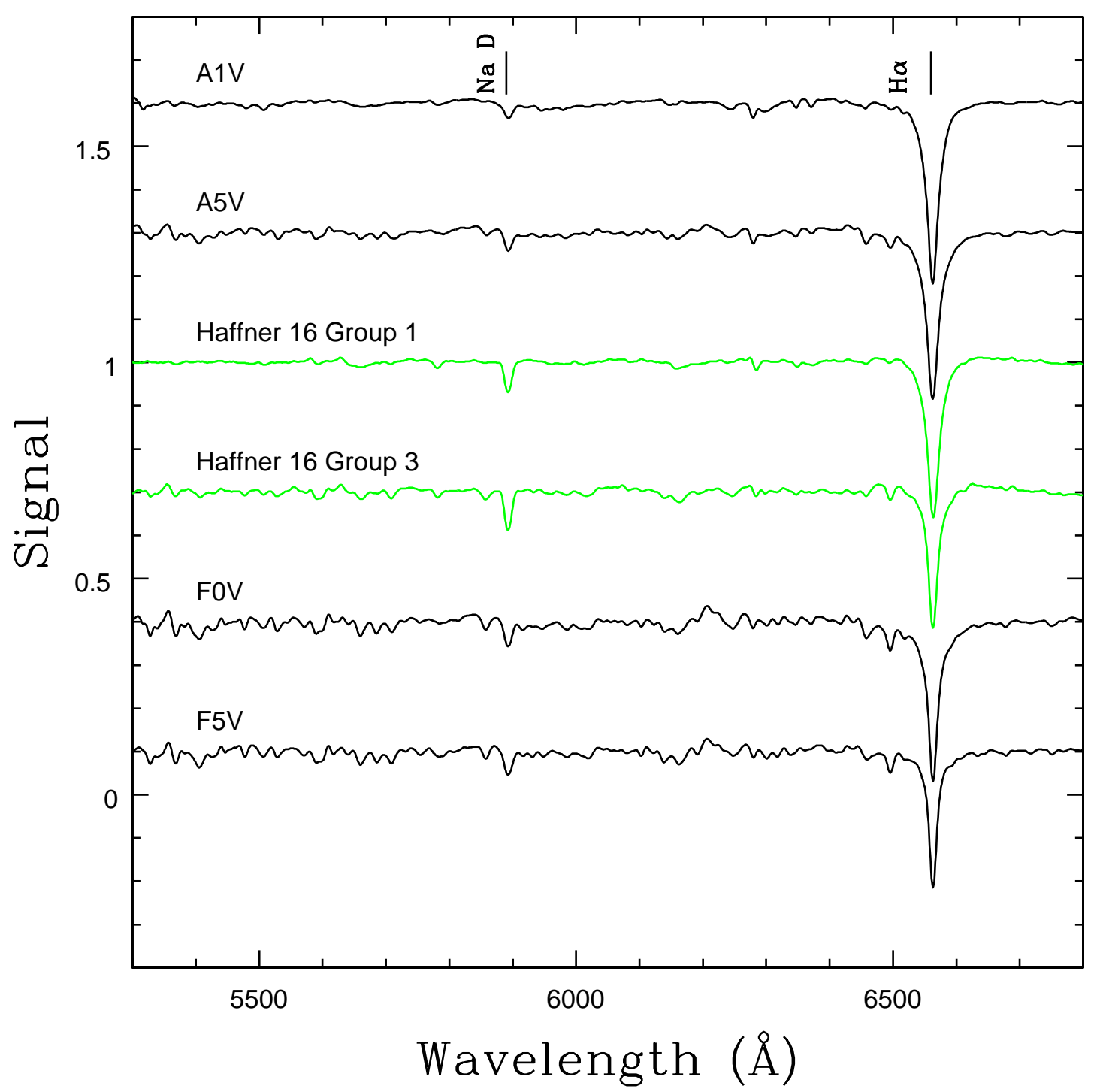

FIG. 14.- Median spectra of stars in groups 1 and 3, which are located near the main sequence on the CMD of Haffner 16. Spectra from the Le Borgne et al. (2003) library are also shown. All spectra have been smoothed to a resolution of 640 and normalized to the continuum. The modest depths of metallic absorption features in the group 1 and 3 spectra are consistent with early to mid-A spectral-types.

left of the cluster center in Figure 1. The wavelength range between 4800 and $5300 \AA$ is of interest as it contains prominent features that probe metallicity and surface gravity, including $\mathrm{H} \beta$, Mgb, and Fe $5270 \AA$. Median spectra of the stars that have $\mathrm{H} \alpha$ in absorption and that have spectra extending down to $4800 \AA$ are shown in Figures 17, 18, and 19. The spectra in these figures have been smoothed to a resolution of 640 and have been normalized to the continuum.

The spectra in Figures 17, 18, and 19 were constructed by combining only a quarter to a third of the total number of objects that were used to construct the spectra in Figures 14, 15, and 16. The comparisons in these figures thus not only provide information about wavelength-related effects but also the sensitivity of the results gleaned at longer wavelengths to sample size. It is thus worth noting that comparisons with the depths of metallic features in the Le Borgne et al. (2003) spectra at blue wavelengths yield characteristic spectral-types that are similar to those found at longer wavelengths. In all five groups $\mathrm{H} \beta$ is also shallower than expected based on the strengths of the metallic features between 4900 and $5300 \AA$ when compared with the Le Borgne et al. spectra. The comparisons involving spectra between 4800 and $5300 \AA$ thus reinforce the results found from spectra at longer wavelengths.

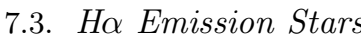

Composite spectra of stars in groups 6 and 7 are shown in Figure 20. There are similarities between the spectra in the wavelength interval $5300-5800 \AA$. However, the group 6 and 7 spectra differ at wavelengths $>5800 \AA$ A. There are differences in the depths of Na D, as well as the Ca I feature at $6170 \AA$. Comparisons with spectra of $\mathrm{K} 2 \mathrm{~V}$ and $\mathrm{K} 7 \mathrm{~V}$ stars from Le Borgne et al. (2003), also shown in Figure 20, suggest that the depths of metallic lines in these spectra are consistent with an early to mid-K spectral type. The characteristic spec- 


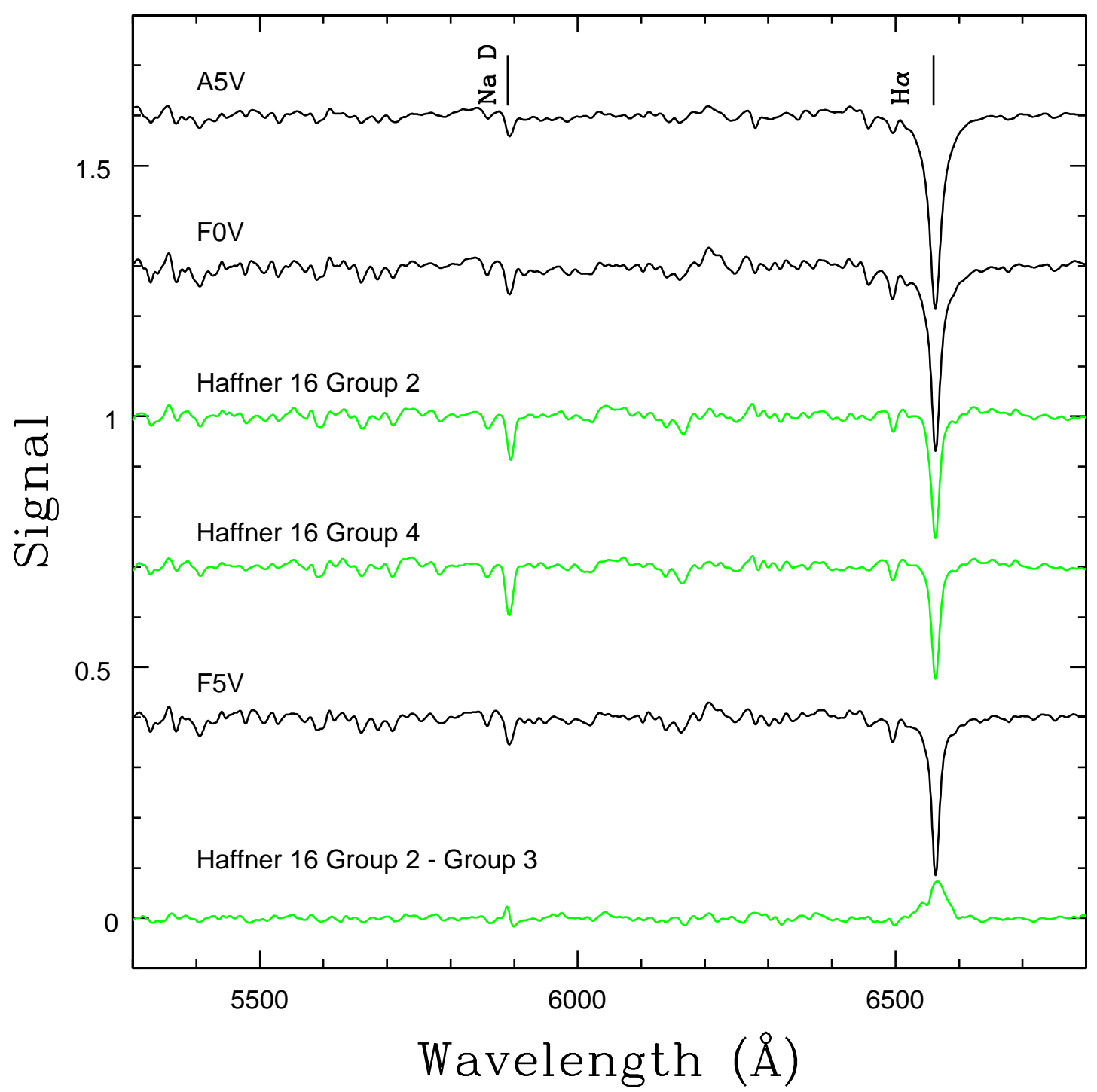

FIG. 15. - Same as Figure 14, but showing the composite spectra of stars in groups 2 and 4. With the exception of minor differences associated with $\mathrm{Na} \mathrm{D}$ and $\mathrm{H} \alpha$, the group 2 and 4 spectra are similar, even though the groups sample stars in different magnitude ranges. The metallic lines in these spectra have depths that are consistent with solar neighborhood stars that have early to mid-F spectral types. $\mathrm{H} \alpha$ has a depth that is weaker than expected based on the depths of metallic lines, and this is attributed to a sub-solar metallicity. The difference between the group 2 and 3 spectra is also shown. There is a tendency for group 2 to have slightly deeper metallic lines.

tral types of the group 6 and 7 spectra were estimated by subtracting various reference star spectra from the group 6 and 7 spectra, and then examining the residuals in the $5400-5800 \AA$ and $6000-6400 \AA$ intervals. The $\mathrm{K} 2 \mathrm{~V}$ reference star gives the best match to the group 6 and 7 spectra.

While the spectral characteristics of groups 6 and 7 are broadly similar to those of group 5 , there are differences, and these are examined in Figure 21, where the results of subtracting the group 5 spectrum from the group 6 and 7 spectra are shown. With the exception of the depth of $\mathrm{Na} \mathrm{D}$, the group 6 spectrum is an overall better match to the group 5 spectrum than the group 7 spectrum. However, the difference in $\mathrm{Na} \mathrm{D}$ depth is such that $\mathrm{Na} \mathrm{D}$ is stronger in group 5 than in group 6 , and the $\mathrm{Na} \mathrm{D}$ line in the group 7 spectrum more closely matches to that in the group 5 spectrum than in group
6. Thus, the strength of $\mathrm{H} \alpha$ emission is not related to $\mathrm{Na} D$ strength.

The mean colors of stars in groups 5,6 , and 7 are not significantly different. Stars in group 7 have a mean $g^{\prime}-i^{\prime}=1.80 \pm 0.06$, whereas the mean for stars in group 6 is $1.79 \pm 0.05$. The uncertainties are the standard error about the mean. The similarity in color makes it unlikely that the differences in the depth of $\mathrm{Na} \mathrm{D}$ between group 6 and 7 is due to circumstellar absorption by a dust-rich component. The equivalent width of $\mathrm{H} \alpha$ emission also appears not to be related to $g^{\prime}-i^{\prime}$ color.

A moderate sub-sample of the stars in group 6 have wavelength coverage that extends to $4800 \AA$, and the median spectrum of sources in group 6 with blue wavelength coverage is shown in Figure 22. Also shown is the spectrum of a $\mathrm{K} 2 \mathrm{~V}$ star and the median spectrum of sources in group 5 from Figure 19. Unfortunately, there 


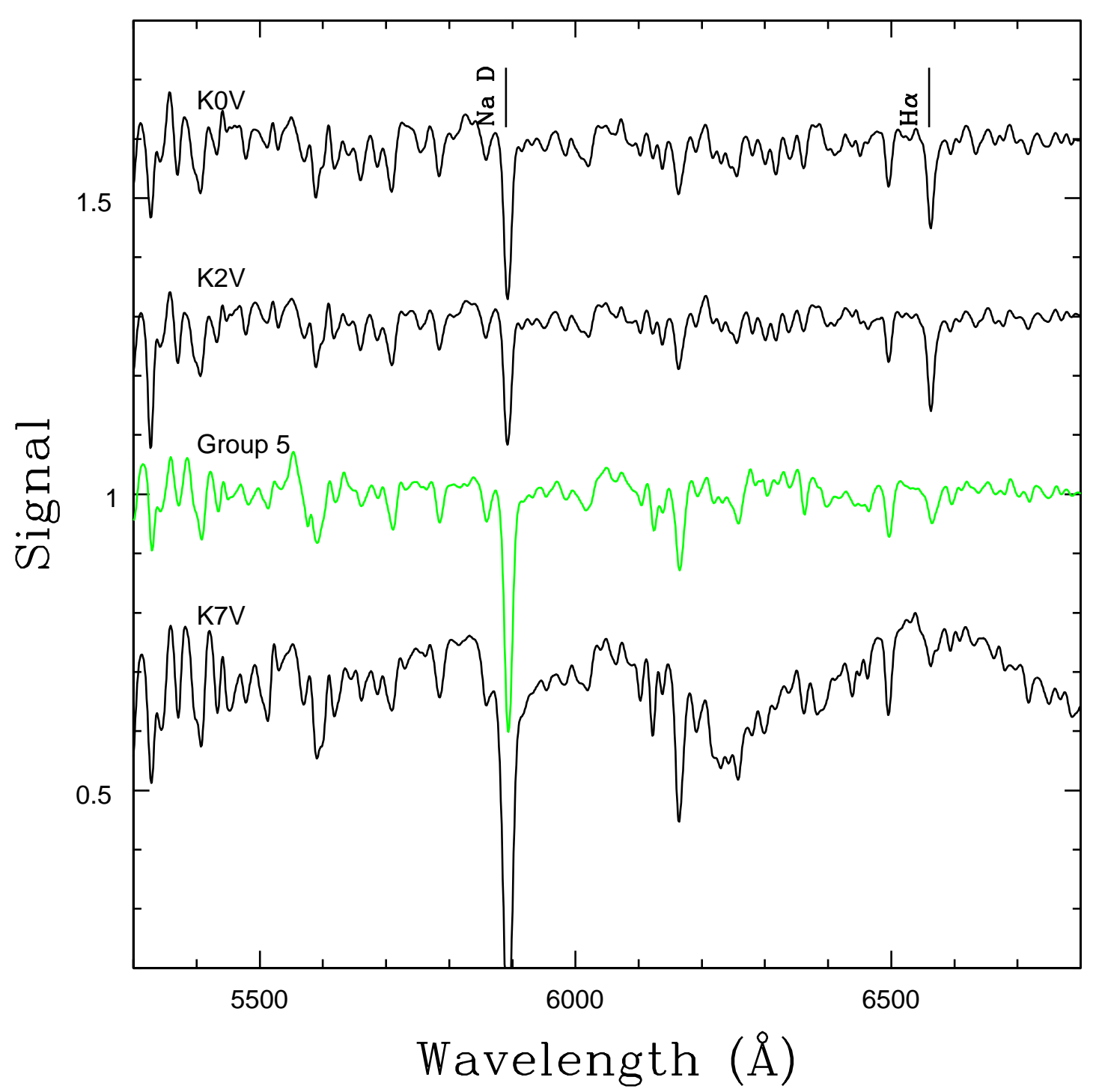

FIG. 16. - Same as Figure 14, but showing stars in group 5. Absorption features in the composite spectrum have depths that are similar to those in the $\mathrm{K} 0 \mathrm{~V}$ and $\mathrm{K} 2 \mathrm{~V}$ spectra.

are not enough stars in Group 7 with wavelength coverage between 4800 and $5300 \AA$ to construct a spectrum with even a moderately high $\mathrm{S} / \mathrm{N}$ ratio.

The difference between the group 6 and group 5 spectra is shown in Figure 22. The scatter in the residuals is larger than in Figure 21. Only a small number of sources have been combined to construct the spectra in Figure 22, and there is lower optical throughput at blue wavelengths than at longer wavelengths. The $\mathrm{S} / \mathrm{N}$ ratio of the blue spectra in Figure 22 is thus lower than at longer wavelengths. There is a possible difference in $\mathrm{H} \beta$ strength between the two groups, although the statistical significance is marginal. This might indicate $\mathrm{H} \beta$ emission in the group 6 spectrum, or could simply indicate a difference in the depth of $\mathrm{H} \beta$ absorption. Fe I 5270 and Mgb both appear to be weaker in the group 6 spectrum than in the group 5 spectrum. The group 6 spectum in Figure 22 is consistent with a $\mathrm{K}$ spectral type, which is in line with the comparisons made at longer wavelengths.

\subsection{Spectra of Four Serendipitous Targets}

The spectra of the four sources that were observed (1) because they could be placed in the same slit as a CMD-selected target, and (2) have locations on the CMD that are distinct from the 7 groups defined above are shown in Figure 23. The locations of 4-21b and 6-02a in the GMOS science field are such that spectra at wavelengths $<5800 \AA$ were not recorded, and so the spectra in Figure 23 are restricted to the wavelength interval 5800 - $6800 \AA$. The feature near $6300 \AA$ in each spectrum is an artifact of [OI] airglow subtraction - these artifacts are not seen in the group $1-7$ spectra as they are suppressed when individual spectra are combined together. The 5-09b spectrum is nulled between 6300 and $6400 \AA$ because this interval samples the gap between CCDs, and the signal was excessively noisy there. Comments on the spectra are as follows:

4-21b: The location of this star on the CMD places it $\sim 0.4$ magnitudes in $g^{\prime}-i^{\prime}$ blueward of the cluster se- 


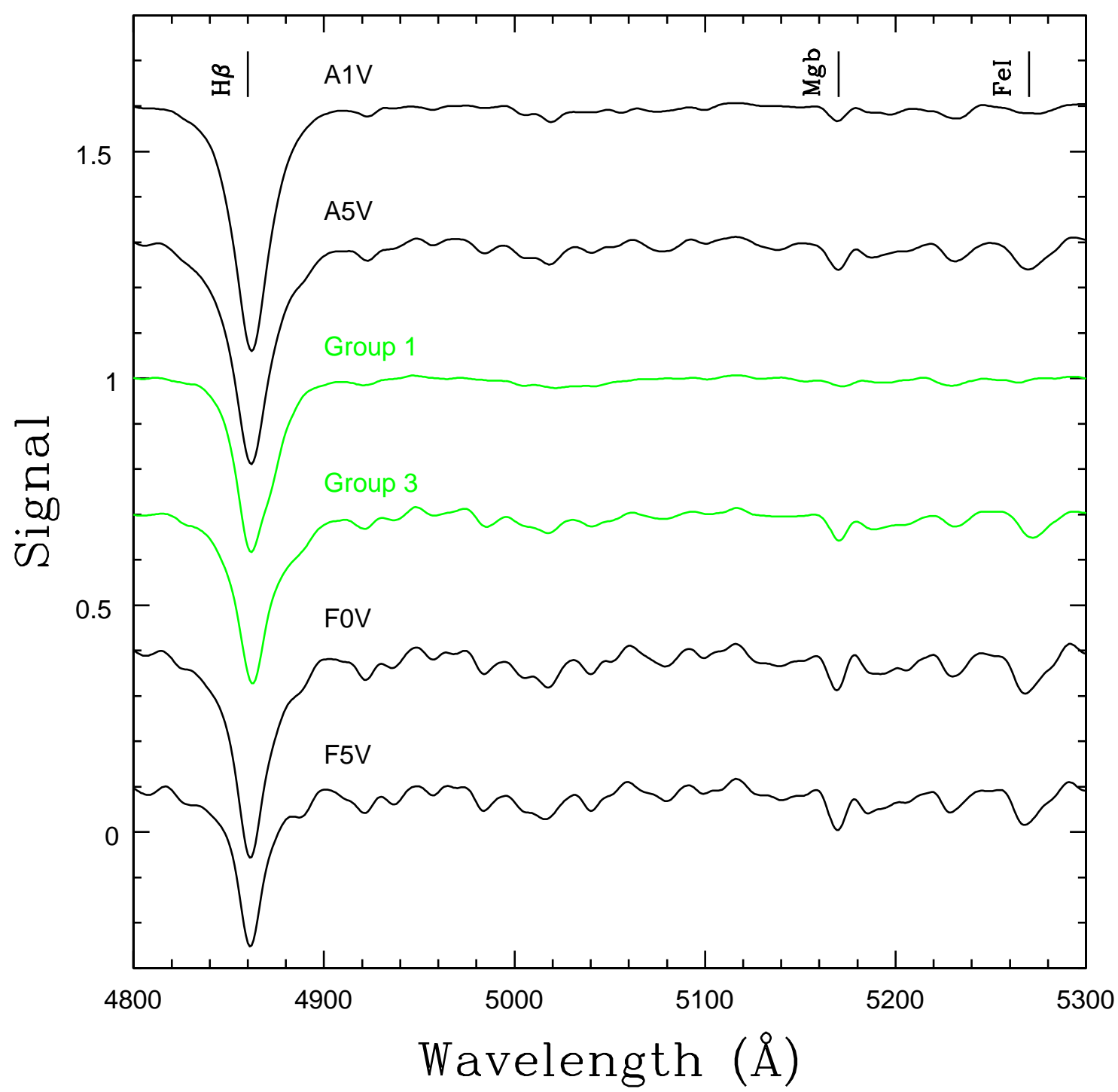

FIG. 17.- Median spectra of stars in groups 1 and 3 with wavelength coverage that extends to $4800 \AA$. Spectra from the Le Borgne et al. (2003) library are also shown. The spectra have been smoothed to a resolution of 640 and normalized to the continuum. The depths of metallic absorption features in the Haffner 16 spectra are consistent with those in early to mid-A spectral types. However, H $\beta$ is shallower than expected for an A-type main sequence star.

quence, close to the near-vertical blue field star sequence. These photometric properties suggest that 4-21b almost certainly does not belong to Haffner 16 . Na D in the 4$21 \mathrm{~b}$ spectrum is deeper than in groups 1 and 3, while $\mathrm{H} \alpha$ is comparable in depth to that in the group 1 spectrum. $4-21 \mathrm{~b}$ is probably a background $\mathrm{F}$ star.

3-05b: The $g^{\prime}-i^{\prime}$ color of star $3-05$ b places it $\sim 0.5$ magnitude redward of the cluster sequence in Figure 10, in a part of the CMD that contains few stars. The weak $\mathrm{H} \alpha$ absorption suggests a late spectral-type, and this is consistent with the deep $\mathrm{Na} \mathrm{D}$ lines. It is likely that $3-05 b$ is a foreground dwarf.

6-02a: Star 6-02a falls close to the cluster sequence in Figure 10. $\mathrm{H} \alpha$ absorption is shallower than in the group 3 spectrum, but is comparable to that in the group 2 and 4 spectra. The $\mathrm{Na} \mathrm{D}$ lines are deeper than in the spectra of groups $1-4$, suggesting a later spectral type than a typical star in these groups. With the exception of $\mathrm{Na} \mathrm{D}$, the metallic lines in the $6-02 \mathrm{a}$ and $3-05 \mathrm{~b}$ spectra have similar depths, and this similarity was confirmed by examining the difference between the two spectra. While the $g^{\prime}-i^{\prime}$ color of $6-02 \mathrm{a}$ is close to that expected for a member of Haffner 16 at this brightness, this star also falls squarely on the foreground dwarf sequence in the CMD. Thus, the relationship between 6-02a and Haffner 16 is uncertain.

5-09b: Star 5-09b is the faintest source for which a spectrum was recorded, and there is considerable noise in the spectrum, even after smoothing. 5-09b falls just redward of the cluster sequence in Figure 10, with an offset in $g^{\prime}-i^{\prime}$ that does not exceed those of stars in groups 5,6 , and 7 . The $\mathrm{Na} \mathrm{D}$ lines are deeper than in the composite spectra of groups 6 and 7, suggesting a later spectraltype than the majority of stars in those groups. The equivalent width of $\mathrm{H} \alpha$ emission in the 5-09b spectrum is by far the largest of any star in the spectroscopic sam- 


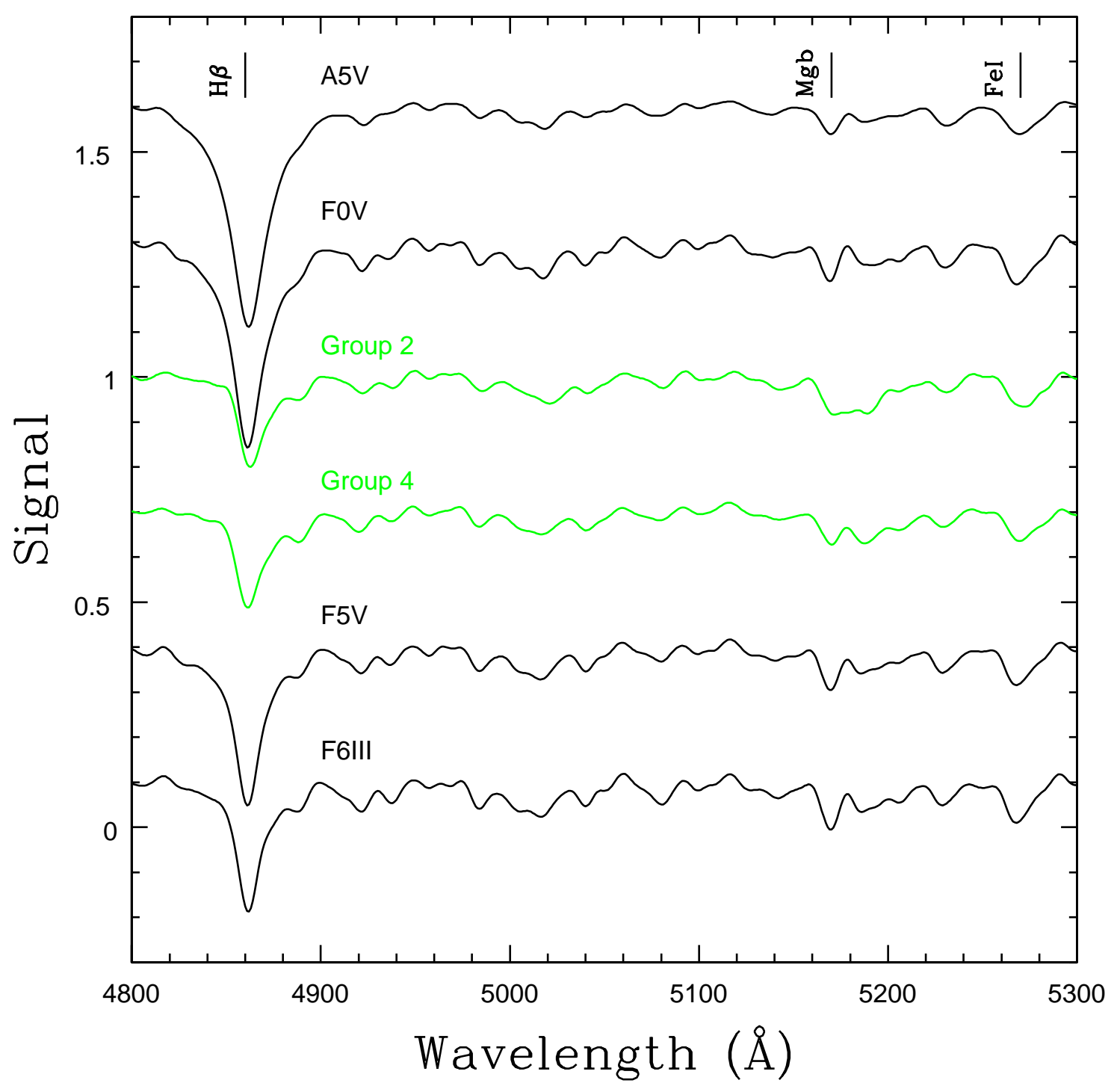

FIG. 18. - Same as Figure 17, but showing the median spectra of the stars in groups 2 and 4 with wavelength coverage extending to $4800 \AA$. As at longer wavelengths, the median spectra of these groups are very similar, with metallic line strengths that match those in solar neighborhood stars that have a late-A or early F spectral type. However, the depth of $\mathrm{H} \beta$ is consistent with later spectral-types.

ple. These characteristics suggest that $5-09 \mathrm{~b}$ is a young $\mathrm{K}$ dwarf, and is a possible PMS star that belongs to Haffner 16 .

\section{DISCUSSION \& SUMMARY}

Images and spectra recorded with GMOS on Gemini South have been used to examine the spectrophotometric properties and spatial distributions of stars in the young open cluster Haffner 16. A tight, well-populated sequence is found in the CMD of objects within 90 arcsec of the cluster center, and this enables the identification of candidate cluster members that span a range of masses. The photometric properties of sub-solar mass stars in Haffner 16 is of particular interest as these objects can provide constraints on the ages of young clusters. Studies of the sub-solar mass regime in young clusters might also provide insights into the photometric properties of PMS stars and thereby set constraints for the models that seek to replicate their properties.
The age estimated for Haffner 16 from the $\left(i^{\prime}, g^{\prime}-\right.$ $\left.i^{\prime}\right)$ CMD and $i^{\prime} \mathrm{LF}$ is older than that found by Davidge et al. (2013) from a deep $(K, J-K)$ CMD and $K$ LF. Both sets of data track the cluster PMS down to masses of a few tenths solar. The difference in age estimates is due to the distance moduli adopted - the Davidge et al. (2013) age of $<10 \mathrm{Myr}$ assumes a distance modulus of 13.5, while the CMD and LF constructed from the present data support an age of 20 Myr with a distance modulus $\sim 12.3$. The age found here is preferable as the visible/red CMD obtained from the GMOS images provides firmer constraints on the distance of Haffner 16 than the NIR CMD. Indeed, structures such as the bend in the CMD of MS stars near $i^{\prime}=15.5$ that serve as anchor points for isochrones are more pronounced at visible wavelengths than in the NIR.

The age found here is older than that estimated by McSwain \& Gies (2005), even though that study assumed a distance modulus of 12.5. The disagreement in age 


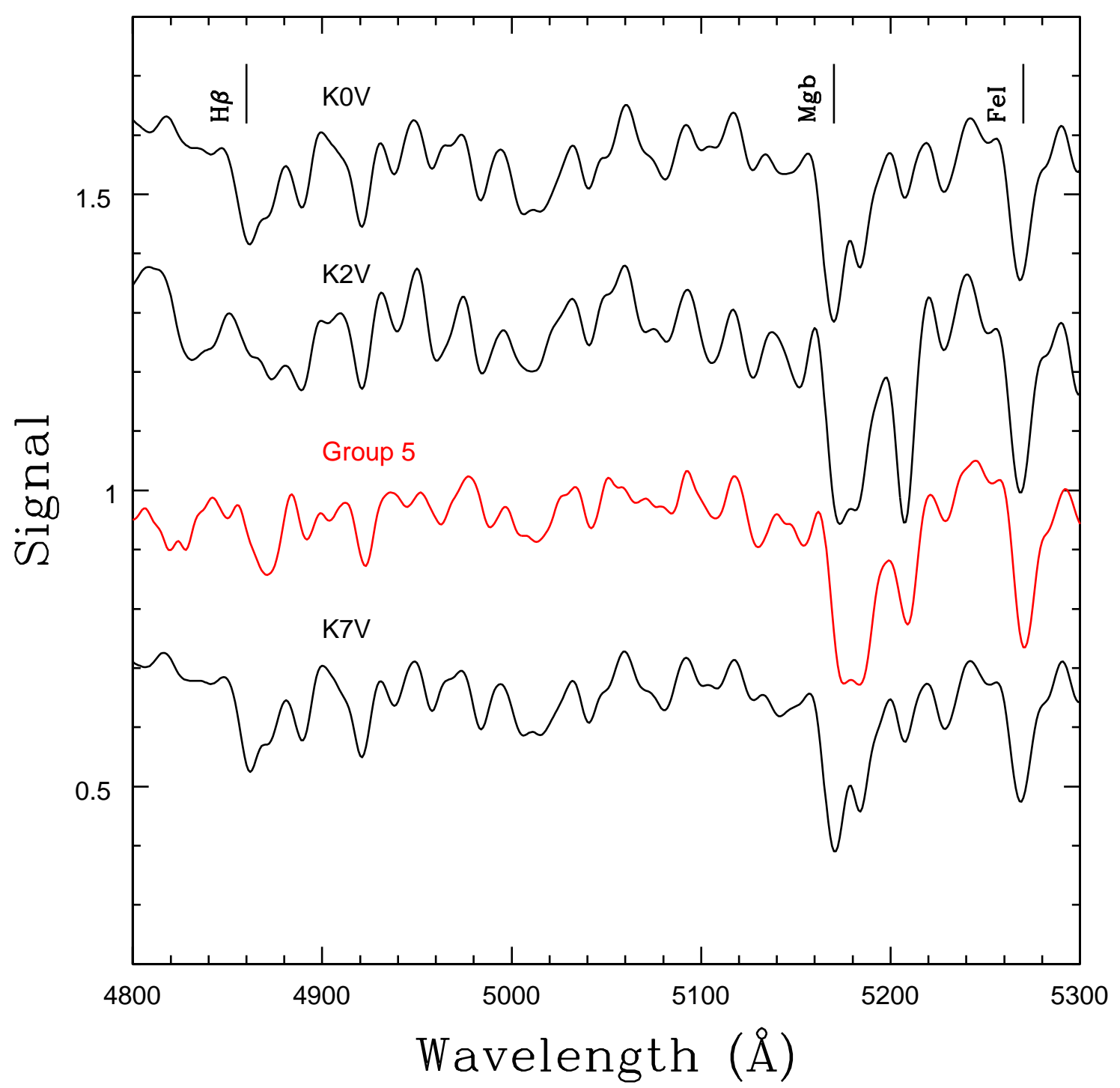

Fig. 19. - Same as Figure 17, but showing stars in group 5. The group 5 spectrum is consistent with a K spectral type.

estimates in likely tied to differences in the area sampled on the sky. The present study considers only objects that are within 1.5 arcmin from the cluster center, which the cluster light profile indicates is an area that is dominated by cluster stars. In contrast, McSwain \& Gies consider objects over a $13.5 \times 13.5$ arcmin area, with the result that there is substantial contamination from field stars. While a modest population of stars related to Haffner 16 may be present in this area (Figure 2), there is no guarantee that these are cluster members or that they formed at the same time as the cluster.

Differences in the age estimates notwithstanding, the line of sight reddening found here agrees with that measured by Davidge et al. (2013). Reddening estimates that are based on the photometric properties of early-type MS stars in Haffner 16 are not sensitive to the adopted distance as these objects define a more-orless vertical trajectory on the visible and NIR CMDs. The agreement between extinction estimates made over a large wavelength range validates the reddening law used in this study, which is the $\mathrm{R}_{V}=3.1$ relation from
Cardelli et al. (1989), as summarized in Table 6 of Schlegel et al. (1998).

Haffner 16 has a well-populated PMS, and so is an interesting laboratory for testing models of PMS evolution. Past studies of young clusters have shown that the agreement between the observed photometric properties of PMS stars and those predicted by models depends on wavelength. Bell et al. (2012) investigated the agreement between models and observations of PMS stars in the Pleiades, which is many tens of Myr older than Haffner 16. While good agreement was found between models and observations in $K$, this was not the case at wavelengths shortward of $1 \mu \mathrm{m}$. Similar behaviour was found by Davidge $(2014 ; 2015)$ when comparing isochrones with the $\left(i^{\prime}, g^{\prime}-i^{\prime}\right)$ and $(K, J-K)$ CMDs of the 20 Myr cluster NGC 2401.

As in the Pleiades and NGC 2401, the level of agreement between the models and observations for Haffner 16 depends on wavelength. Davidge et al. (2013) found that solar metallicity isochrones matched the location of PMS stars in Haffner 16 on the $(K, J-K)$ CMD 


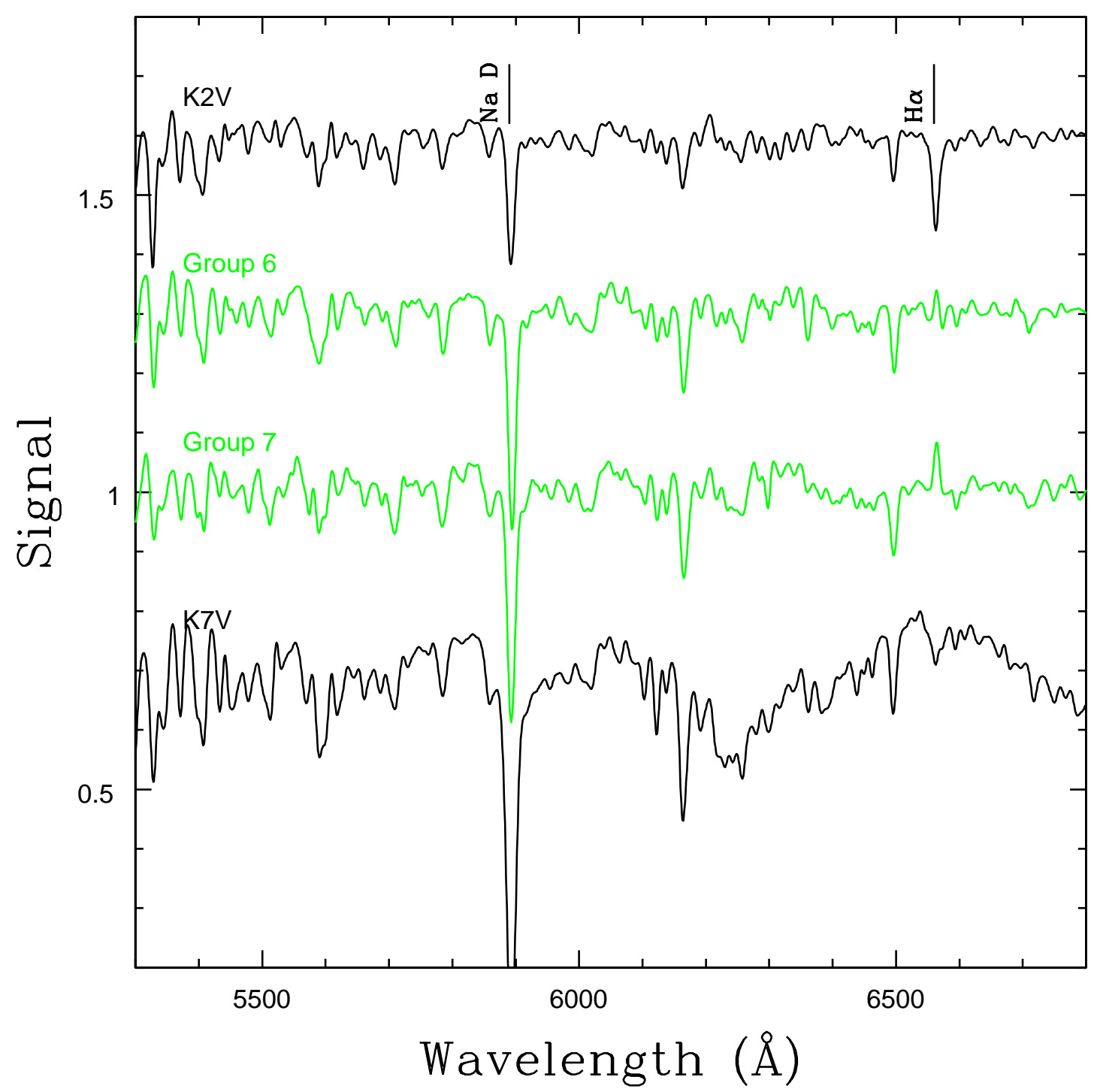

FIG. 20.- Median spectra of stars in groups 6 and 7 are compared with spectra of stars from the Le Borgne et al. (2003) library. Na $\mathrm{D}$ and $\mathrm{Ca}$ I $6170 \AA$ in the group 7 (stars with pronounced $\mathrm{H} \alpha$ emission) spectrum are deeper than in the group 6 (stars with weak H $\alpha$ emission) spectrum. There are also subtle differences between the group 6 and 7 spectra at wavelengths $>6200 \AA$. The behaviour of H $\alpha$ aside, the depths of metallic features in both groups are consistent with an early to mid-K spectral-type.

over the full range of magnitudes sampled. The PMS sequence in the NIR CMD is almost vertical, and so is not sensitive to the adopted distance modulus. However, in Section 5 it is shown that the same isochrones do not track the PMS sequence on the $\left(i^{\prime}, g^{\prime}-i^{\prime}\right)$ CMD, falling progressively blueward of cluster stars when $i^{\prime}<18$.

What is the cause of the offset between the models and the Haffner 16 sequence on the $\left(i^{\prime}, g^{\prime}-i^{\prime}\right)$ CMD? The presence of line emission among PMS stars in Haffner 16 may provide clues to answering this question. Davidge et al. (2013) discuss narrow-band images that reveal possible $\operatorname{Br} \gamma$ emission among stars that have $K>15$ in Haffner 16. If $\mathrm{Br} \gamma$ emission is present then prominent $\mathrm{H} \alpha$ emission should also be seen among faint stars in Haffner 16, and the GMOS spectra confirm that this is the case. That the majority of $\mathrm{H} \alpha$ emission sources tend to be found within or near the Shoulder region (Figure 12 ) is consistent with them being cluster members.
$\mathrm{Br} \gamma$ and $\mathrm{H} \alpha$ emission are signatures of chromospheric activity. Such activity among PMS stars in Haffner 16 might occur if these objects are accreting material. Obscuration by circumstellar material might then explain the red colors of PMS stars, although this would require a remarkable alignment of accretion disks along the line of sight, coupled with a level of obscuration that increases towards low masses. However, accretion is an unlikely explanation for line emission in Haffner 16, as the time scale for the decay of stellar accretion disks is a few Myr (e.g. Haisch et al. 2001; Fedele et al. 2010, but see also De Marchi et al. 2011; 2013), whereas Haffner 16 has an age of $\sim 20$ Myr.

Strong emission lines and associated continuum emission will affect broad-band colors. However, line and continuum emission do not affect the $g^{\prime}-i^{\prime}$ colors of PMS stars in Haffner 16 by a significant amount. Evidence for this comes from the $g^{\prime}-i^{\prime}$ colors of the stars in groups 


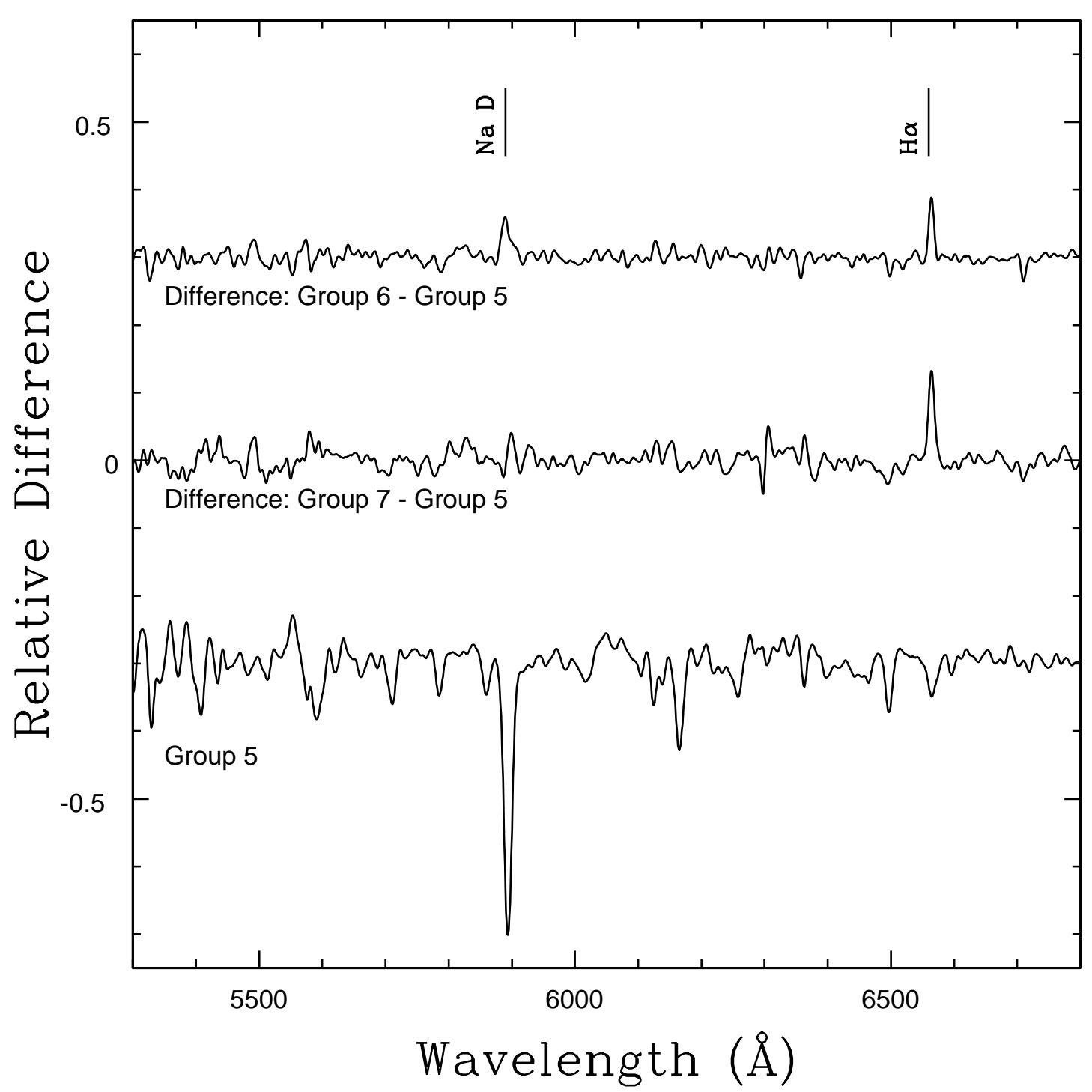

FIG. 21.- Differences between the spectra of the two groups with $\mathrm{H} \alpha$ in emission and the group 5 spectrum. The group 5 spectrum from Figure 16 is shown to assist with line identifications. With the exception of $\mathrm{Na} \mathrm{D}$, the residuals indicate that the group 5 spectrum is better matched by the group 6 spectrum than the group 7 spectrum. Any differences in line strength due to circumstellar absorption must be modest given the similar mean $g^{\prime}-i^{\prime}$ colors of stars in the groups (see text).

5,6 , and 7 . The mean colors of stars in these groups are similar, even though they have very different $\mathrm{H} \alpha$ characteristics (Section 7.3). The similar metallic line strengths seen in the composite spectra of these groups also suggests that line veiling - which will occur if there is continuum emission - is not significant in the stars that have $\mathrm{H} \alpha$ in emission.

Star spots with temperatures that are cooler than the surrounding photosphere are a possible explanation of the offets between the models and observations. Spot activity can be driven by rapid rotation in young stars, and has been proposed to explain the SED of low mass stars in the Pleiades (e.g. Stauffer et al. 2003). Somers \& Pinsonneault (2015) examine the influence that cool star spots may have on the photometric properties of PMS stars. They consider spots that have properties similar to those seen in actual stars, and investigate the affect that spots have on photometric properties with respect to stellar mass (and hence effective temperature). Their models show that light from stars at visible wavelengths is susceptible to spot activity as this is where the SEDs of spots peak; in contrast, the NIR part of the spectrum is little changed. Star spots can thus explain qualitatively the offsets between isochrones and the Haffner 16 PMS at visible wavelengths, and the agreement between observations and theory in the NIR.

The majority of candidate PMS stars with spectra in this study have $i^{\prime} \sim 18$, and there is only a small offset between the isochrones and observed PMS sequence at this magnitude (e.g. Figure 5). While $\mathrm{H} \alpha$ emission is seen at this brightness, it has a modest strength. If spots affect the broad-band colors of PMS stars in Haffner 16 then spectra of fainter (and thus lower mass) PMS stars in this cluster should show more pronounced $\mathrm{H} \alpha$ emission, that correlates with the difference in $g^{\prime}-i^{\prime}$ between the isochrones and observations. This expectation is con- 


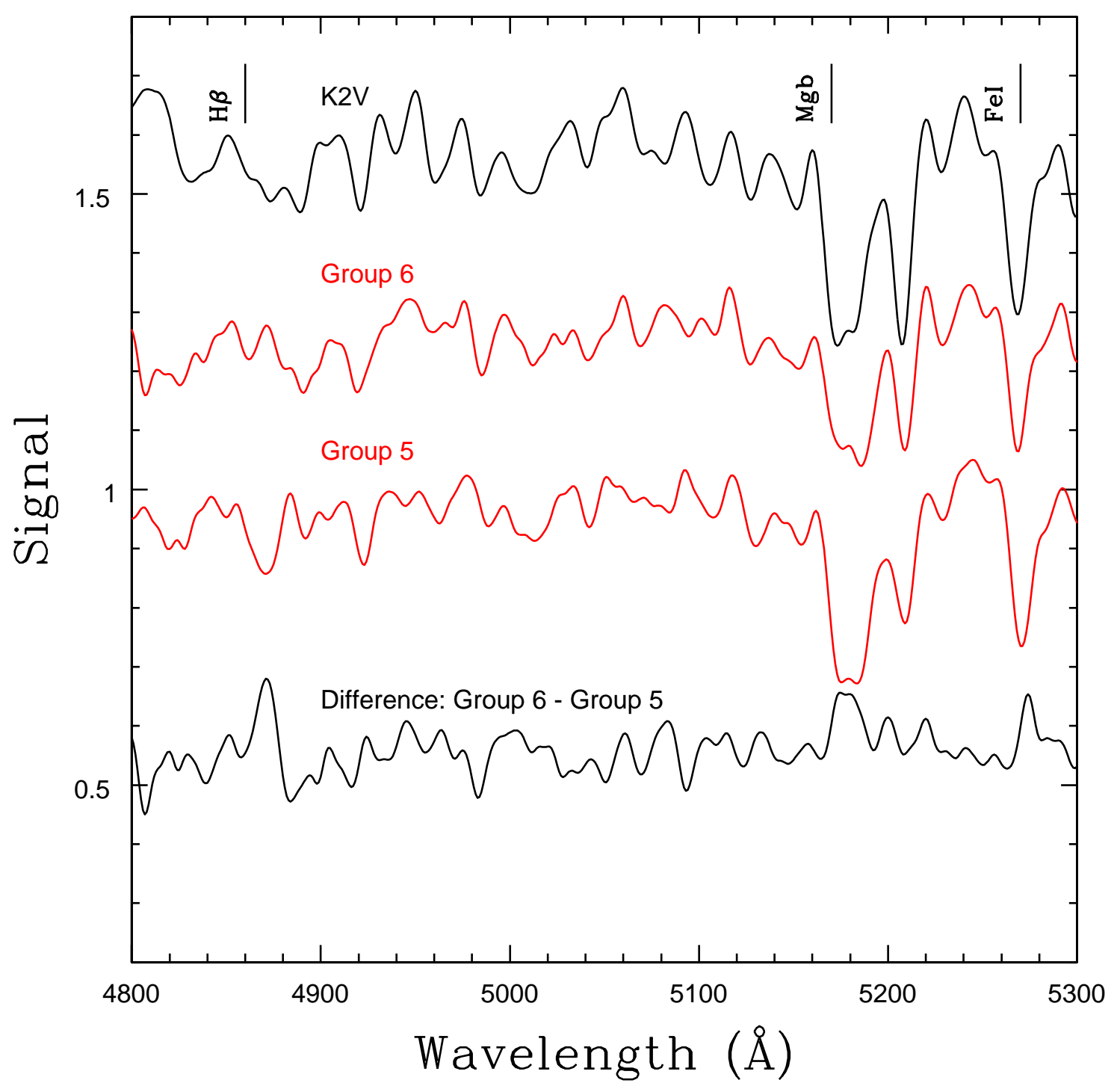

FIG. 22.- Spectra of groups 5 and 6 at blue wavelengths. The spectrum of a K2V star from the Le Borgne et al. (2003) library is also shown. The difference between the group 5 and 6 spectra shows larger residuals than when the spectra at longer wavelengths are compared. This is due in part to smaller sample sizes and the comparatively low system throughput at wavelengths $<5300 \AA$. Still, there are indications that absorption features in group 6 at these wavelengths are weaker than those in group 5.

sistent with the level of $\mathrm{H} \alpha$ emission seen in the spectrum of $5-09 \mathrm{~b}$, which is the faintest source with a spectrum in this study, and is also the object in the current sample with the largest equivalent width of $\mathrm{H} \alpha$ emission. Spectra or narrow-band $\mathrm{H} \alpha$ images of sources with $i^{\prime}>19$ in Haffner 16 will reveal if the spectroscopic properties of $5-09 \mathrm{~b}$ are typical of faint stars in the cluster. If spots do affect the photometric properties of PMS stars in Haffner 16 then high dispersion spectra should also reveal these stars to be rapid rotators. The moderately tight nature of the PMS in the CMD would require that the majority of cluster stars be spotted. By extension, spectroscopic and/or narrow-band observations of PMS stars in the 20 Myr old cluster NGC 2401 - which have photometric properties that are similar to their counterparts in Haffner 16 - should also show evidence for prominent $\mathrm{H} \alpha$ emission.

It is a pleasure to thank the anonymous referee for providing timely and comprehensive reports that greatly improved the manuscript.

\section{REFERENCES}

Balser, D. S., Rood, R. T., Bania, T. M., \& Anderson, L. D. 2011, ApJ, 738, 27

Bell, C. P. M., Naylor, T., Mayne, N. J., Jeffries, R. D., \& Littlefair, S. P. 2012, MNRAS, 424,3178

Bonnell, I. A., Smith, R. J., Clark, P. C., \& Bate, M. R. 2011, MNRAS, 410, 2339

Bressan, A., Marigo, P., Girardi, L., et al. 2012, MNRAS, 427, 127 Cardelli, J. A., Clayton, G. C., \& Mathis, J. S. 1989, ApJ, 345, 245
Chabrier, G. 2003, PASP, 115, 763

Daflon, S., \& Cunha, K. 2004, ApJ, 617, 1115

Davidge, T. J., Carrasco, E. R., Winge, C., Pessev, P., Neichel, B., Vidal, F., \& Rigaut, F. 2013, PASP, 125, 1181

Davidge, T. J., Andersen, D. R., Lardière, O., et al. 2016, AJ, 152, 173

Davidge, T.J. 2012, ApJ, 761, 155

Davidge, T. J. 2014, ApJ, 781, 95 


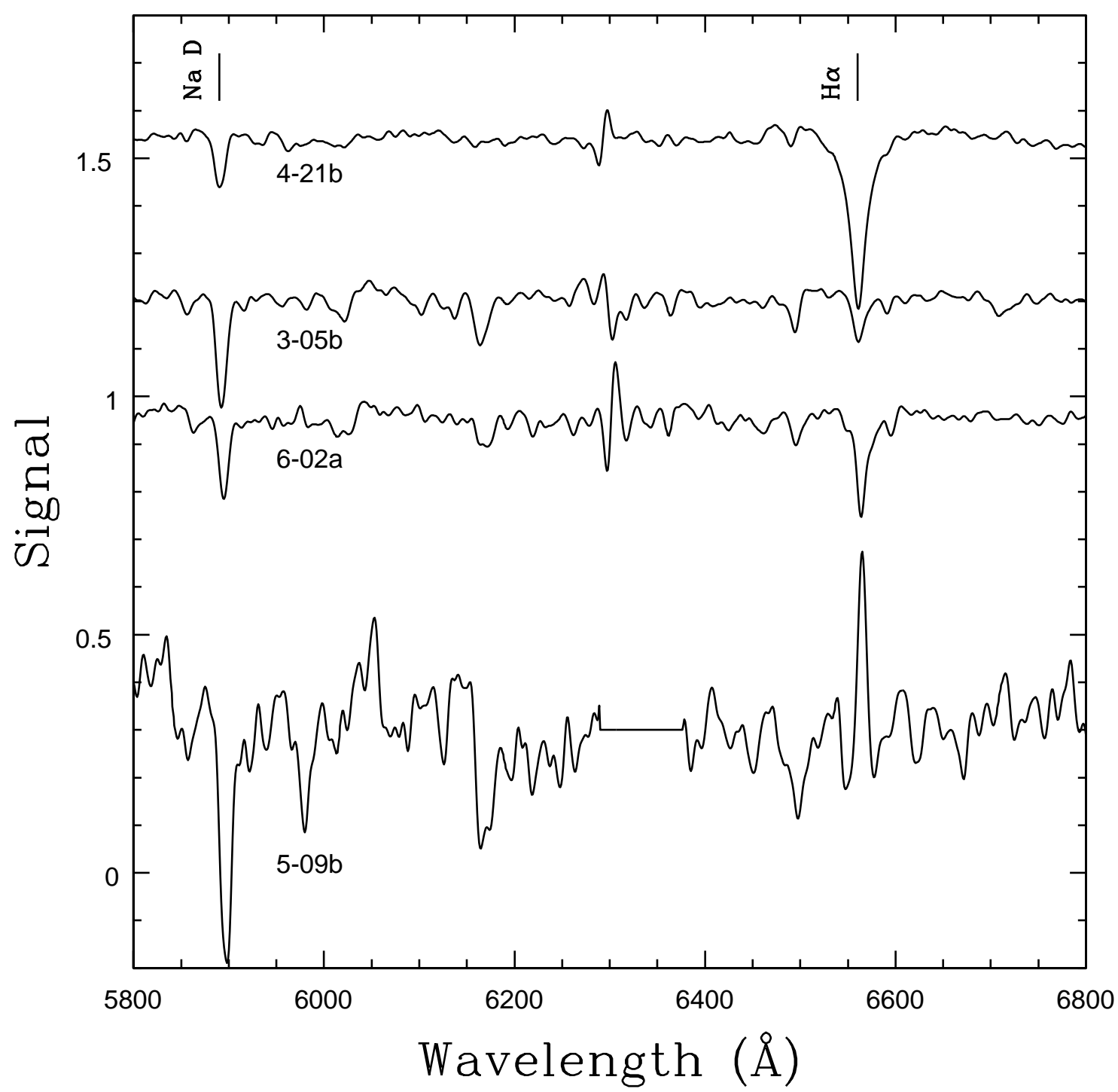

FIG. 23. - Spectra of four stars in the serendipitous sample. The locations of these stars on the CMD are indicated in Figure 10. The spectra have been smoothed to a resolution of 640 , and normalized to the continuum. The noise feature near $6300 \AA$ in each spectrum is an artifact of [OI] airglow emission. Star 5-09b is the faintest object in the spectroscopic sample, and the region that is nulled between 6300 and $6400 \AA$ in its spectrum is the gap between CCDs, where the $\mathrm{S} / \mathrm{N}$ ratio is poor. It is argued in the text that $4-21 \mathrm{~b}$ and $3-05 \mathrm{~b}$ are probably not cluster members, while the membership of 6-02a is uncertain. However, the photometric and spectroscopic properties of 5-09b suggests that it may belong to Haffner 16.

Davidge, T. J. 2015, PASP, 127, 836

Davidge, T. J. 2017, ApJ, 837, 178

de Grijs, R., Goodwin, S. P., \& Anders, P. 2013, MNRAS, 436, 136

De Marchi, G., Panagia, N., Guarcello, M. G., \& Bonito, R. 2013, MNRAS, 435, 3058

De Marchi, G., Panagia, N., Romaniello, M., et al. 2011, ApJ, 740, 11

Fall, S. M., \& Chandar, R. 2012, ApJ, 752, 96

Fedele, D., van den Ancker, M. E., Henning, T., Jayawardhana, R., \& Oliveira, J. M. 2010, A\&A, 510, A72

Fujii, M. S., \& Portegies Zwart, S. 2016, ApJ, 817, 4

Haisch, K. E., Jr., Lada, E. A., \& Lada, C. J. 2001, ApJ, 553, L153

Hayes, C. R., Friel, E. D., Slack, T. J., \& Boberg, O. M. 2015, AJ, 150,200

Hook, I. M., Jorgensen, I., Allington-Smith, J. R., Davies, R. L., Metcalfe, N., Murowinski, R. G., \& Crampton, D. 2004, PASP, 116,425

Jarrett, T. H., Cohen, M., Masci, F., et al. 2011, ApJ, 735, 112

Kruijssen, J. M. D., Maschberger, T., Moeckel, N., et al. 2012, MNRAS, 419, 841
Lada, C. J., \& Lada, E. A. 2003, ARA\&A, 41, 57

Le Borgne, J.-F., Bruzual, G., Pello, R., et al. 2003, A\&A, 402, 433 Matzner, C. D., \& Jumper, P. H. 2015, ApJ, 815, 68

McSwain, M. V., \& Gies, D. R. 2005, ApJS, 161, 118

Pecaut, M. J., \& Mamajek, E. E. 2013, ApJS, 208, A9

Pfalzner, S., Parmentier, G., Steinhausen, M., Vincke, K., \& Menten, K. 2014, ApJ, 794, 147

Pfalzner, S. Vincke, K., \& Xiang, M. 2015, A\&A, 576, A28

Poznanski, D., Prochaska, J. X., \& Bloom, J. S. 2012, MNRAS, 426, 1465

Schlegel, D. J., Finkbeiner, D. P., \& Davis, M. 1998, ApJ, 500, 525

Silva-Villa, E., Adamo, A., Bastian, N., Fouesneau, M., \& Zackrisson, E. 2014, MNRAS, 440, L116

Somers, G., \& Pinnsonneault, M. H. 2015, ApJ, 807, 174

Stauffer, J. R., Jones, B. F., Backman, D., Hartmann, L. W., Barrado Y Navascues, D., Pinsonneault, M. H., Terndrup, D. M., \& Muench, A. A. 2003, AJ, 126, 833

Stetson, P. B. 1987, PASP, 99, 191

Stetson, P. B., \& Harris, W. E. 1988, AJ, 96, 909

Vogt, N., Moffat, A. F. J. 1972, A\&AS, 7, 133 
Wallace, L., Hinkle, K. H., Livingston, W. C., \& Davis, S. P. 2011,

ApJS, 195, A6
Worthy, G., Faber, S. M., Gonzalez, J. J., \& Burstein, D. 1994, ApJS, 94, 687

Wright, E. L., Eisenhardt, P. R. M., Mainzer, A. K., et al. 2010, AJ, 140, 1868

TABLE 4

Spectroscopic Targets

\begin{tabular}{|c|c|c|c|c|c|c|}
\hline ID & $\mathrm{RA}$ & Dec & $\mathrm{x}$ & $\mathrm{y}$ & $i^{\prime}$ & $g^{\prime}-i^{\prime}$ \\
\hline $1-01^{*}(2-01)$ & 117.543569 & -25.473770 & 2038.52 & 195.710 & 14.591 & -0.042 \\
\hline $1-02 *(2-02)$ & 117.561257 & -25.483789 & 2285.60 & 589.080 & 14.825 & 0.186 \\
\hline 1-03 & 117.566457 & -25.483040 & 2267.18 & 705.049 & 14.596 & 0.123 \\
\hline $1-04$ & 117.574332 & -25.495831 & 2582.41 & 879.969 & 15.025 & 0.313 \\
\hline $1-05^{*}(2-05)$ & 117.577887 & -25.443279 & 1287.63 & 959.210 & 14.087 & -0.140 \\
\hline 1-06 & 117.582393 & -25.451210 & 1483.15 & 1059.30 & 14.264 & -0.038 \\
\hline $1-07$ & 117.585096 & -25.466511 & 1860.14 & 1120.01 & 14.425 & 0.007 \\
\hline $1-08$ & 117.588058 & -25.456129 & 1604.39 & 1185.65 & 15.006 & 0.050 \\
\hline $1-09 *(2-09)$ & 117.592056 & -25.478230 & 2149.06 & 1274.56 & 14.662 & -0.124 \\
\hline $1-10 *(2-10)$ & 117.594252 & -25.450649 & 1469.45 & 1323.00 & 13.650 & -0.137 \\
\hline $1-11 *(2-11)$ & 117.597878 & -25.459641 & 1691.00 & 1404.01 & 14.571 & -0.092 \\
\hline $1-12$ & 117.600510 & -25.478060 & 2144.87 & 1462.51 & 15.024 & -0.140 \\
\hline $1-13^{*}(2-13)$ & 117.604551 & -25.452379 & 1512.29 & 1552.50 & 14.831 & 0.518 \\
\hline $1-14^{*}(2-14)$ & 117.615952 & -25.475849 & 2090.76 & 1806.00 & 14.323 & -0.257 \\
\hline $1-15^{*}(2-15)$ & 117.619822 & -25.433611 & 1050.01 & 1892.39 & 14.543 & 0.759 \\
\hline $1-16 *(2-16)$ & 117.630665 & -25.449350 & 1437.84 & 2134.00 & 14.186 & 0.617 \\
\hline $2-01 *(1-01)$ & 117.543569 & -25.473770 & 2038.52 & 195.710 & 14.591 & -0.042 \\
\hline $2-02 *(1-02)$ & 117.561257 & -25.483789 & 2285.60 & 589.080 & 14.825 & 0.186 \\
\hline 2-03 & 117.567780 & -25.452490 & 1514.44 & 734.239 & 15.015 & 0.035 \\
\hline $2-04$ & 117.572773 & -25.497339 & 2619.65 & 845.320 & 14.906 & 0.442 \\
\hline $2-05^{*}(1-05)$ & 117.577887 & -25.443279 & 1287.63 & 959.210 & 14.087 & -0.140 \\
\hline $2-06$ & 117.583258 & -25.458920 & 1672.97 & 1078.65 & 14.803 & 0.210 \\
\hline $2-07$ & 117.586255 & -25.457600 & 1640.48 & 1145.40 & 14.810 & -0.120 \\
\hline $2-08$ & 117.588887 & -25.439720 & 1200.13 & 1204.06 & 14.639 & 0.821 \\
\hline $2-09 *(1-09)$ & 117.592056 & -25.478230 & 2149.06 & 1274.56 & 14.662 & -0.124 \\
\hline $2-10 *(1-10)$ & 117.594252 & -25.450649 & 1469.45 & 1323.00 & 13.650 & -0.137 \\
\hline $2-11 *(1-11)$ & 117.597878 & -25.459641 & 1691.00 & 1404.01 & 14.571 & -0.092 \\
\hline $2-12$ & 117.601111 & -25.468460 & 1908.41 & 1475.93 & 14.342 & 0.576 \\
\hline $2-13^{*}(1-13)$ & 117.604551 & -25.452379 & 1512.29 & 1552.50 & 14.831 & 0.518 \\
\hline $2-14^{*}(1-14)$ & 117.615952 & -25.475849 & 2090.76 & 1806.00 & 14.323 & -0.257 \\
\hline $2-15^{*}(1-15)$ & 117.619822 & -25.433611 & 1050.01 & 1892.39 & 14.543 & 0.759 \\
\hline $2-16 *(1-16)$ & 117.630665 & -25.449350 & 1437.84 & 2134.00 & 14.186 & 0.617 \\
\hline $3-01 *(4-01)$ & 117.544506 & -25.468800 & 1915.97 & 216.179 & 15.472 & 0.658 \\
\hline 3-02 & 117.549083 & -25.436319 & 1115.77 & 317.829 & 15.287 & 0.807 \\
\hline 3-03 & 117.553968 & -25.488550 & 2402.67 & 426.859 & 15.426 & 0.654 \\
\hline $3-04^{*}(4-04)$ & 117.556415 & -25.449940 & 1451.59 & 481.209 & 15.420 & 0.927 \\
\hline 3-05a & 117.558746 & -25.461929 & 1747.00 & 533.239 & 15.700 & 0.390 \\
\hline 3-05b & 117.559249 & -25.461905 & 1747.60 & 550.700 & 16.847 & 1.424 \\
\hline $3-06 *(4-06)$ & 117.561121 & -25.449490 & 1440.56 & 585.820 & 15.961 & 0.702 \\
\hline $3-07 *(4-07)$ & 117.563975 & -25.453800 & 1546.66 & 649.270 & 15.734 & 0.476 \\
\hline $3-08$ & 117.567451 & -25.429319 & 943.650 & 726.770 & 15.904 & 0.824 \\
\hline $3-09 *(4-09)$ & 117.570255 & -25.452900 & 1524.51 & 789.250 & 16.015 & 0.542 \\
\hline 3-10 & 117.575862 & -25.471861 & 1991.70 & 914.030 & 15.194 & 0.577 \\
\hline $3-11$ & 117.580469 & -25.452280 & 1509.52 & 1016.55 & 15.571 & 0.596 \\
\hline $3-12$ & 117.587435 & -25.445601 & 1344.93 & 1171.42 & 15.159 & 0.619 \\
\hline $3-13$ & 117.591949 & -25.429119 & 938.969 & 1272.19 & 15.328 & 0.813 \\
\hline 3-14 & 117.595253 & -25.456421 & 1611.64 & 1345.44 & 15.630 & 0.634 \\
\hline $3-15$ & 117.599487 & -25.435949 & 1107.33 & 1440.01 & 15.081 & 0.781 \\
\hline $3-16^{*}(4-17)$ & 117.603507 & -25.454220 & 1557.44 & 1529.28 & 15.872 & 0.420 \\
\hline $3-17 *(4-18)$ & 117.605939 & -25.449539 & 1442.39 & 1583.72 & 15.680 & 0.369 \\
\hline $3-18$ & 117.610395 & -25.429110 & 938.880 & 1683.15 & 15.278 & 0.674 \\
\hline $3-19^{*}(4-20)$ & 117.613478 & -25.439489 & 1194.70 & 1751.76 & 15.960 & 0.712 \\
\hline 3-20 & 117.624078 & -25.442789 & 1276.21 & 1987.28 & 15.261 & 0.661 \\
\hline $3-21$ & 117.629099 & -25.429670 & 953.049 & 2099.37 & 15.736 & 0.497 \\
\hline $3-22^{*}(4-23)$ & 117.633748 & -25.483009 & 2267.17 & 2202.25 & 15.491 & 0.444 \\
\hline $4-01 *(3-01)$ & 117.544506 & -25.468800 & 1915.97 & 216.179 & 15.472 & 0.658 \\
\hline 4-02 & 117.548947 & -25.436581 & 1122.16 & 315.220 & 15.813 & 0.828 \\
\hline 4-03 & 117.553067 & -25.461161 & 1727.81 & 406.920 & 15.976 & 0.581 \\
\hline $4-04^{*}(3-04)$ & 117.556415 & -25.449940 & 1451.59 & 481.209 & 15.420 & 0.927 \\
\hline 4-05 & 117.558961 & -25.462400 & 1758.43 & 537.789 & 16.026 & 0.779 \\
\hline $4-06 *(3-06)$ & 117.561121 & -25.449490 & 1440.56 & 585.820 & 15.961 & 0.702 \\
\hline $4-07 *(3-07)$ & 117.563975 & -25.453800 & 1546.66 & 649.270 & 15.734 & 0.476 \\
\hline 4-08 & 117.566943 & -25.488420 & 2399.71 & 715.469 & 15.274 & 0.542 \\
\hline $4-09 *(3-09)$ & 117.570255 & -25.452900 & 1524.51 & 789.250 & 16.015 & 0.542 \\
\hline 4-10 & 117.577264 & -25.457500 & 1637.97 & 945.270 & 15.956 & 0.679 \\
\hline $4-11 a$ & 117.581971 & -25.452629 & 1518.19 & 1049.93 & 15.532 & 0.868 \\
\hline $4-11 b$ & 117.580473 & -25.452248 & 1509.52 & 1016.56 & 15.571 & 0.596 \\
\hline 4-12 & 117.585704 & -25.469730 & 1939.42 & 1133.10 & 15.383 & 0.361 \\
\hline $4-13 a$ & 117.588186 & -25.474010 & 2045.04 & 1188.56 & 15.475 & 0.935 \\
\hline $4-13 \mathrm{~b}$ & 117.588623 & -25.474132 & 2048.10 & 1198.28 & 15.695 & 0.708 \\
\hline $4-14 a$ & 117.590911 & -25.451870 & 1499.50 & 1249.11 & 15.888 & 0.423 \\
\hline
\end{tabular}


TABLE 4

Spectroscopic Targets

\begin{tabular}{|c|c|c|c|c|c|c|}
\hline $4-14 b$ & 117.591291 & -25.451637 & 1493.77 & 1257.58 & 16.310 & 0.603 \\
\hline 4-15 & 117.593422 & -25.431339 & 993.710 & 1305.04 & 15.612 & 0.877 \\
\hline $4-16$ & 117.600982 & -25.482161 & 2245.90 & 1472.66 & 15.475 & 0.560 \\
\hline $4-17^{*}(3-16)$ & 117.603507 & -25.454220 & 1557.44 & 1529.28 & 15.872 & 0.420 \\
\hline $4-18 *(3-17)$ & 117.605939 & -25.449539 & 1442.39 & 1583.72 & 15.680 & 0.368 \\
\hline $4-19 *(5-15,6-07)$ & 117.611318 & -25.436760 & 1127.38 & 1703.61 & 15.817 & 0.504 \\
\hline $4-20 *(3-19)$ & 117.613478 & -25.439489 & 1194.70 & 1751.76 & 15.960 & 0.712 \\
\hline $4-21 a$ & 117.624779 & -25.485121 & 2319.12 & 2002.35 & 15.255 & 0.439 \\
\hline $4-21 b$ & 117.625425 & -25.484931 & 2314.45 & 2016.80 & 16.869 & 0.370 \\
\hline $4-22^{*}(5-19,6-10)$ & 117.629156 & -25.445040 & 1331.68 & 2100.31 & 15.105 & 0.656 \\
\hline $4-23^{*}(3-22)$ & 117.633748 & -25.483009 & 2267.17 & 2202.25 & 15.491 & .444 \\
\hline $5-01 *(6-01)$ & 117.633748 & -25.483009 & 2507.56 & 243.380 & 15.376 & 0.866 \\
\hline 5-02 & 117.552874 & -25.432360 & 1018.42 & 402.420 & 18.737 & 1.728 \\
\hline $5-03$ & 117.554998 & -25.450260 & 1459.31 & 449.829 & 18.998 & .888 \\
\hline $5-04$ & & -25.4 & & & & 613 \\
\hline $5-05$ & 117.566049 & -25.470680 & 1962.70 & & & 834 \\
\hline $5-06$ & 99346 & 61491 & 1736.31 & & 029 & 846 \\
\hline $5-07$ & 2458 & -25 & & & & 043 \\
\hline $5-08$ & & 6030 & & & 486 & 642 \\
\hline $5-09 a$ & & -25 . & & & & 755 \\
\hline $5-09 b$ & 3932 & -25 & & 982.310 & & 2.602 \\
\hline $5-10$ & & -25. & & & & \\
\hline & 117 & -25 . & & & & 682 \\
\hline & & & & & & 613 \\
\hline $5-13$ & 1511 & -25 . & & & & 1.960 \\
\hline $5-14 a$ & & -25.4 & & & 18.905 & 2.036 \\
\hline $5-14 b$ & 117.6 & -25.4 & $104 \varepsilon$ & 1562.03 & & \\
\hline $5-15^{*}(4-19,6-07)$ & 117.6 & -25.4 & 1127.38 & 1703.61 & 15.817 & 0.504 \\
\hline 5-16 & & -25 . & & & & 55 \\
\hline $5-17$ & 117.6 & -25.4 & 198 & 1974.27 & 763 & 1.645 \\
\hline $5-18$ & & & & & & 1.701 \\
\hline $19 *(4-22,6-10)$ & 156 & -25.4 & 133 & 2100 & 15.105 & 0.656 \\
\hline $5-20$ & 117.6 & -25. & 1751.65 & 2164.44 & 18.477 & 1.646 \\
\hline $5-21$ & & & & & & 1.939 \\
\hline $6-01 *(5-01)$ & 117.545714 & -25.492781 & 2507.56 & 243.380 & 67 & 0.866 \\
\hline 6-02a & & & & & & 0.934 \\
\hline $6-02 b$ & & -25 & & & & 1.882 \\
\hline $6-03$ & 117. & -25.4 & 190 & & & 1.757 \\
\hline $6-04 a$ & & & & & & \\
\hline $6-04 b$ & 117.5 & -25.4 & 1255. & & & 1.794 \\
\hline & & & & & & 2.064 \\
\hline & & -25.455910 & 1599 & 1543.75 & 18.957 & 1.981 \\
\hline $6-07 *(4-19,5-15)$ & 117.611318 & -25.4367 & 1127.38 & 1703.61 & 15.8 & 0.504 \\
\hline & & & & & & \\
\hline $6-0$ & & -25.482040 & & & & 1.670 \\
\hline $6-10 *(4-22,5-19)$ & 117.629156 & -25.445040 & 1331.68 & 2100.31 & 15.105 & 0.656 \\
\hline
\end{tabular}

